\title{
Value-at-risk and extreme value distributions for financial returns
}

\section{Konstantinos Tolikas}

Cardiff Business School, Cardiff University, Aberconway Building, Colum Drive, Cardiff, CF10 3EU, UK; email: TolikasK@cardiff.ac.uk

\begin{abstract}
The ability of the generalized extreme value (GEV) and generalized logistic $(G L)$ distributions to fit extreme financial returns in the stock, commodities and bond markets is assessed. The empirical results indicate that the too much celebrated GEV is not the most appropriate model for the data since the fatter tailed GL is found to provide better descriptions of the extreme returns. Extreme value theory (EVT) based value-at-risk (VaR) estimates are then derived and compared with those generated by traditional methods. The results show that when the focus is on the really ruinous events, which are located deep into the tails of the returns distribution, the EVT methods used in this study can be particularly useful since they produce VaR estimates that outperform those derived by the traditional methods at high confidence levels. However, these estimates were found to be considerably higher than those derived by traditional VaR models, consequently leading to higher capital reserves for financial institutions.
\end{abstract}

\section{INTRODUCTION}

Value-at-risk (VaR) is the maximum potential loss of a portfolio over a particular time horizon at a certain confidence level. During the last decade it has become an industry standard and it is now routinely used by financial firms when estimating a capital cushion against potential financial catastrophes; indeed, it is known as the minimum capital requirement (MCR). Statistically, VaR is defined as one of the lower quantiles of the distribution of returns that is only exceeded by a certain probability (eg, $5 \%$ or $1 \%$ ). Therefore, it is argued that accurate VaR estimates imply accurate descriptions of the tails of the distribution of financial returns. A convenient assumption usually made is that returns follow a normal distribution. However, a large amount of empirical research suggests that the actual distribution of returns has a fatter lower tail than that suggested by the normal. ${ }^{1}$ One implication of this feature is that the probability of large losses is much greater than implied by the normal distribution. In such a case, VaR models

Konstantinos Tolikas would like to thank J. R. M. Hosking, IBM Research Division, T. J. Watson Research Center, for kindly providing the codes for the estimation of the standard errors of the parameter estimates.

${ }^{1}$ See Aparicio and Estrada (2001) for a review of the literature on the empirical distribution of financial returns. 
are prone to fail when they are needed most, ie, where a financial institution may suffer enormous losses because of an extreme fall in share prices.

A branch of statistics, named extreme value theory (EVT), focuses exclusively on these extremes and their associated probabilities by directly studying the tails of probability distributions. Applications in finance include, among others, Longin (1996) who investigated the limiting distribution of extremes in the US stock market, Lux (2001) who applied EVT to German data, Jondeau and Rockinger (2003) who analyzed the daily extreme returns of 27 stock markets and Gençay and Selçuk (2004) who applied EVT to emerging markets. They all found that extremes of financial returns could be adequately characterized by the Fréchet distribution (a member of the generalized extreme value (GEV) family). The role of EVT as an input in VaR estimation has been examined by Danielson (2002) who used US data to compare daily VaR estimates at the $99 \%$ confidence level derived from the variance-covariance (VC), historical simulation (HS), generalized autoregressive conditional heteroskedasticity $(\mathrm{GARCH})$, exponential weighted moving average (EWMA) and EVT methods. He found that the EVTbased VaR provides more accurate estimates than all the other models. Pownall and Koedijk (1999) used data from Asian stock markets to compared VaR estimates generated by the normal distribution and the RiskMetrics model of JP Morgan with estimates generated using EVT. They found that the EVT-based VaR significantly outperformed the other two models and attributed this to the ability of EVT to fit fat-tailed time series. Similar results were obtained by Neftci (2000) for the case of eight major exchange and interest rates. He also found that EVT-based VaR estimates were $20 \%$ to $30 \%$ larger than those generated by the normal distribution. Bali (2003) used daily observations of the annualized yield of the three-month, six-month, one-year and 10-year US treasury securities from 1954 to 1998 . He rejected the normality hypothesis and found that the GEV and generalized Pareto (GP) distributions could lead to very precise VaR results. He also found that EVT-based VaR estimates were on average 24\% to $38 \%$ larger than those generated by the normal distribution. Based on this finding, he argued that the multiplication factor that the Bank for International Settlements (Basel Committee on Banking Supervision (1996)) uses to adjust the VaR estimates of banks, which employ their own internal models, is rather too high and should be reduced.

The literature that explores EVT applications in finance has a number of similarities. Firstly, in most studies the GEV and GP are the only distributions used to fit the extremes. Secondly, the maximum likelihood parameter estimation method is mainly used. A notable exception is Gettinby et al (2004) who investigated the distribution of extreme share returns in the UK from 1975 to 2000 and found that the generalized logistic (GL) distribution describes both the minima and maxima data better than the GEV. Another exception is Da Silva and Mendes (2003) who used probability weighted moments (PWM) to estimate the parameters of the limiting distribution of extremes in 10 Asian stock markets. However, they focused solely on the GEV distribution that was found to provide an adequate fit to the data. Recently, Tolikas and Brown (2006) considered the 
GL, GEV and GP distributions and investigated the distribution of the extreme daily share returns in the Greek stock market. Their results added further support to the ability of the GL to fit extreme data and illustrated that the GL provides more accurate VaR estimates compared to the GEV and the normal distribution. Therefore, there are reasons to believe that there is scope for improvement and this is what this paper attempts to do by employing EVT methods whose use in finance has not yet been fully investigated.

The first aim of this paper is to describe the distribution of the extreme minima for daily returns of a wide set of markets and instruments; this set includes the French stock market, the US commodities market and the German bond market. ${ }^{2}$ The second aim of this paper is to assess whether this EVT approach can be useful for risk measurement purposes by deriving VaR estimates and comparing to those generated by traditional approaches. The remainder of the paper is set out as follows. Section 2 introduces the EVT methodology adopted in this paper; Section 3 describes the data and Section 4 contains the results of the analysis of the extremes; in Section 5, VaR estimates generated by the EVT and traditional approaches are presented and compared; Section 6 discuses the implications of the results for both regulators and financial institutions and Section 7 concludes the paper.

\section{APPLYING EXTREME VALUE THEORY TO ESTIMATE VALUE-AT-RISK}

EVT is the statistical study of the extremal behavior of random variables and its role is to develop procedures which are scientifically appropriate for describing and estimating their behavior. ${ }^{3}$ Extremes of financial returns are defined as the minimum of the daily (or weekly, monthly or larger time periods) logarithmic returns over a given period (known as the selection interval). To illustrate this point, let us denote the time series of an index daily log-returns with the variable $Y_{1}, Y_{2}, \ldots, Y_{n}$. If the length of the selection interval is $m$, we divide the series into non-overlapping time intervals of length $m$. The time series of the extreme minima will be $X_{1}=\min \left(Y_{1}, \ldots, Y_{m}\right), X_{2}=\min \left(Y_{m+1}, \ldots, Y_{2 m}\right), \ldots, X_{n / m}=$ $\min \left(Y_{n-m}, \ldots, Y_{n}\right)$. The problem is then to find a probability distribution that adequately describes their behavior. VaR estimates can then be calculated as certain lower quantiles of this distribution. Applying EVT to financial data involves a number of steps. Firstly, the length of the minima selection period must be chosen. Secondly, distributions that are likely to model adequately the empirical extreme minima returns should be identified. Thirdly, the parameters of these distributions should be estimated and the goodness-of-fit of these distributions to the data

\footnotetext{
${ }^{2}$ The focus is kept on describing the lower tail of the returns distribution since this is where the big losses of a long position are located. However, similar analysis can be applied to the upper tail for the case of a short position.

${ }^{3}$ For a thorough introduction to EVT the reader is advised to see Coles (2001) and Embrechts et al (1999).
} 
should be tested to choose the one that best fits the empirical data. In the following paragraphs these steps are analytically presented.

The number of extremes available for analysis depends on the length of the extremes selection interval. A longer interval will result in fewer extremes and thus, a lower level of efficiency when estimating a distribution's parameters. To some extent this is an arbitrary decision and in this paper it was decided to use extremes defined over weekly time spans (five trading days). ${ }^{4}$ The behavior of the extremes distribution over time aggregation is also studied by dividing the series of weekly extremes into 10 and 30 subperiods.

Under the assumption that returns are independent and identically distributed (iid), first Fisher and Tippet (1928) and later Gnedenko (1943) showed that the limiting distribution of the extremes ought to be the GEV. In particular, the EVT indicates that the limiting distribution of the maxima (and minima) collected over non-overlapping time periods of equal length ought to be, after being normalized and centered, one of the three distributions that make up the GEV family: the Gumbell, Fréchet and Weibull. An alternative way to analyze the behavior of the extremes is known as the peaks over threshold (POT) method, according to which extremes are defined as excesses over a threshold. Balkema and de Haan (1974) and Pickands (1975) showed that the limiting distribution of excesses over a high enough threshold ought to be the GP. The main advantage of the GP over the GEV is considered to be the more efficient use of data since by collecting the extremes as excesses over a threshold more data is left in the tails. Consequently, the GP is considered to be a good alternative to the GEV distribution when modeling extreme financial data; see, for example, Cotter and Dowd (2006) and Bali (2003). A crucial issue, however, is the right choice of the threshold: a low one will result in many central observations entering the sample while a high one will leave so few in the sample that it could lead to inaccurate estimates. In addition, the asymptotic theory of extremes is based on the assumption that extremes are serially independent or at most weakly dependent. However, financial returns tend to cluster and this could lead to considerable serial dependence in the time series of the extremes. Regrettably, there is a decision to be made and in order to avoid or reduce as much as possible the problem of serial dependence in the time series of extremes it was decided to collect the extremes as the minimum daily returns over non-overlapping time intervals of prespecified length. It should also be noted that EVT focuses exclusively on the tails of the returns distribution and says nothing about the central part that can be fitted better by other distributions (eg, normal). In addition, as noted by Gilli and Këllezi (2006), financial returns are not always fat tailed and in this case other distributions, including the normal, may be found to adequately fit the whole distribution.

\footnotetext{
${ }^{4}$ Monthly (20 trading days) and quarterly (60 trading days) selection intervals were also employed but the results were not very different from those reported here. However, from the VaR estimation point of view, weekly extremes are preferable since they result in more frequent updating of the distributions parameters. Hence, the results for the monthly and quarterly minima are not included in this paper.
} 
The GEV is a three parameter distribution and its probability density function (pdf) is given by:

$$
\begin{aligned}
f(x) & =\alpha^{-1} \mathrm{e}^{-(1-\kappa) y} \mathrm{e}^{-\mathrm{e}^{-y}} \\
\text { where } y & = \begin{cases}-\kappa^{-1} \log \{1-\kappa(x-\beta) / \alpha\}, & \kappa \neq 0 \\
(x-\beta) / \alpha, & \kappa=0\end{cases}
\end{aligned}
$$

the parameters $\alpha, \beta$ and $\kappa$ are called the scale, location and shape, respectively. The first parameter is analogous to the standard deviation and high values imply that the distribution of extremes is widely spread out while the second is analogous to the mean and high values imply large extremes. The third governs the shape of the distribution and it is probably the most important parameter since larger values correspond to fatter-tailed distributions. The Weibull distribution is the special case of the GEV when $\kappa>0$ and the range of $x$ is $-\infty<x \leq \beta+\alpha / \kappa$. The Gumbel distribution is obtained for $\kappa=0$ and the range of $x$ is $-\infty<x<\infty$, while when $\kappa<0$ the Fréchet distribution is obtained and the range of $x$ is $\beta+\alpha / \kappa \leq x<\infty$. The cumulative distribution function (cdf), $F(x)$, and the quantile function, $X(F)$, of a GEV distributed variable $X$ are given in Appendix A (together with their counterparts for the GL distribution).

However, although the GEV enjoys theoretical support there is strong evidence that financial returns exhibit heteroscedasticity and serial correlation. Kearns and Pagan (1997) used simulations to show that the shape parameter estimates can be exaggerated when the iid assumption is violated. On the other hand, Leadbetter et al (1983) showed that EVT is valid for data structures with weak dependence. With respect to VaR estimation the series of the data will be divided into subperiods and moving window techniques will be used to estimate the parameters. This can be reasonably assumed to capture some of the non-stationarity of the data thus, reducing the non-iid data problem. Another alternative would be to fit the tail of the conditional distribution of returns by using an autoregressive volatility model (eg, GARCH), standardize the returns by the estimated conditional volatility and proceed in EVT analysis. This approach has received attention by McNeil and Frey (2000) and Byström (2004). However, additional parameters have to be estimated that make this approach subject to increased estimation error and model risk. Typically, a conditional risk measure will react after the event and it will assume that the high-risk period will continue leading to high-risk measures. In that respect, from a financial institution's point of view an unconditional approach might be preferable because it will not lead to frequent changes in traders' portfolios when risk limits are reached. Alternatively, the non-constant variance of returns feature would tend to diminish if lowerfrequency data were to be used. However, the size of the dataset will also decrease, significantly raising concerns for the soundness of the estimation procedures. Therefore, the iid assumption was relaxed but at the same time the GL distribution was also included, accepting a trade off between being theoretically correct and empirically convincing. 
The pdf of the GL distribution is given by:

$$
\begin{gathered}
f(x)=\alpha^{-1} \mathrm{e}^{-(1-\kappa) y} /\left(1+\mathrm{e}^{-y}\right)^{2} \\
\text { where } y= \begin{cases}-\kappa^{-1} \log \{1-\kappa(x-\beta) / \alpha\}, & \kappa \neq 0 \\
(x-\beta) / \alpha, & \kappa=0\end{cases}
\end{gathered}
$$

The logistic distribution is the special case of the GL when $\kappa=0$ and $x$ is in the range $-\infty<x<\infty$, while when $\kappa>0, x$ belongs to $-\infty<x \leq \beta+\alpha / \kappa$ and when $\kappa<0, x$ belongs to $\beta+\alpha / \kappa \leq x<\infty$.

The detection of the best candidate distributions to fit the data is accomplished using L-moment diagrams. L-moments are linear combinations of ordered data which, like the conventional moments, provide a set of summary statistics for probability distributions. Hosking (1990) defined the $r$ th L-moment, $\lambda_{r}$, for any random variable $X$, which has a finite mean, as:

$$
\lambda_{r} \equiv r^{-1} \sum_{\kappa=0}^{r-1}(-1)^{\kappa}\left(\begin{array}{c}
r-1 \\
\kappa
\end{array}\right) \mathrm{E} X_{(r-\kappa: r)}, \quad r=1,2, \ldots
$$

where $\mathrm{E} X_{(r-\kappa: r)}$ is the expectation of the $(r-\kappa)$ th extreme order statistic. The first two such statistics, $\lambda_{1}$ and $\lambda_{2}$, are measures of location and scale and the two L-moment ratios, $\tau_{3}=\lambda_{3} / \lambda_{2}$ and $\tau_{4}=\lambda_{4} / \lambda_{2}$ are measures of skewness and kurtosis, respectively. The most important feature of the L-moments is that they are more robust to the presence of outliers than conventional moments; this is because the calculations of conventional moments involve powers, which give greater weight to outliers, that can lead to considerable bias and variance in the parameter and quantiles estimators. In addition, Hosking (1990) showed that for samples of more than 20 data points the asymptotic biases of the L-moments are negligible for most distributions. Finally, sample L-moments can take any value that the population moments can take: this is not true for conventional moments because they have algebraic bounds. An L-moment diagram contains the curves or points of the theoretical distributions whose ability to adequately fit the empirical data is examined. ${ }^{5}$ The identification of the best candidate distributions is achieved by plotting the estimated $\tau_{3}$ and $\tau_{4}$ and choosing the distribution whose L-skewness and L-kurtosis theoretical curve is closest to the plotted point.

The next step is to estimate the parameters of the selected distribution(s). For moderate to large samples, the most widely used method is the maximum

\footnotetext{
${ }^{5}$ On such a diagram, a three-parameter distribution (eg, the GL) is represented by a curve whereas a two-parameter distribution (eg, the normal) is represented by a single point. The idea behind the L-moment ratio diagrams is similar to that of the Karl Pearson system of curves (Karl Pearson used the coefficients of skewness and kurtosis to compare the shapes of various distributions used in hydrology), in that it allows the visual assessment of many probability distributions in a single diagram. The usefulness of L-moment ratio diagrams in identifying candidate distributions for the empirical data is well documented and illustrated in the literature. See, for example, Vogel and Fennessey (1993), Hosking (1990) and Sankarasubramanian and Srinivasan (1999).
} 
likelihood method. However, its asymptotic properties are open to doubt in the case of small samples where convergence of the likelihood function is not always guaranteed to be at the global maximum (Hill (1963)). For small samples, which are the norm in EVT, the PWM is considered to generate more unbiased parameter and quantile estimates than the maximum likelihood, though is no more efficient. Hosking et al (1985) showed that for the GEV distribution, parameters and quantiles made using the PWM method are estimated with at least $70 \%$ efficiency. For example, when the shape parameter of the GEV is -0.2 , the asymptotic bias of the 0.01 quantile estimated by the PWM and maximum likelihood methods is found to be -0.2 and 1.6, respectively. In addition, for shape parameter values in the range -0.5 to 0.5 and samples of up to 100 observations, PWM estimates have lower root-mean square error than estimates generated by the maximum likelihood method. Similar results are reported in the literature for the GEV (Landwehr et al (1979); Smith (1987)) and for the GP (Hosking and Wallis (1987); Roótzen and Tajvidi (1997)). Hosking (1990) defined the PWM of a random variable $X$ with a finite mean and a distribution function $F$ as:

$$
\beta_{r}=E\left\lfloor X\{F(X)\}^{r}\right\rfloor, \quad r=0,1, \ldots
$$

where $E[X(\cdot)]$ is the expectation of the quantile function of $X$. Although, PWM may be sensitive to outliers, Hosking (1990) demonstrated that there exist linear relationships between the PWM and the more robust L-moments, given by:

$$
\lambda_{r+1}=\sum_{k=0}^{r} P_{r, k}^{*} \beta_{k}, \quad r=0,1, \ldots, \quad \text { where } P_{r, k}^{*}=(-1)^{r-k}\left(\begin{array}{c}
r \\
k
\end{array}\right)\left(\begin{array}{c}
r+k \\
k
\end{array}\right)
$$

This method involves estimating parameters by equating sample moments to those of the chosen distribution. For the GEV and GL the solutions for the shape $(\kappa)$, scale $(\alpha)$ and location $(\beta)$ parameter estimates can be found in Appendix A.

After fitting a distribution, it is important to assess how good the fit is. For this reason the Anderson-Darling goodness-of-fit test is used (Anderson and Darling (1954)). This is a test especially designated to measure discrepancies in the tails between the empirical and theoretical distribution function and it has been found to be the most powerful among a wide set of available tests for small samples (Choulakian and Stephens (2001); Stephens (1976)). A tractable expression is given in d'Agostino and Stephens (1986):

$$
A_{n}^{2}=-n-(1 / n) \sum_{i=1}^{n}\left[(2 i-1) \log z_{i}+(2 n+1-2 i) \log \left(1-z_{i}\right)\right]
$$

where $z_{i}=F\left(x_{i}\right), i=1, \ldots, n$, is the empirical distribution function of a variable $X$ of size $n$.

Once the empirical distribution of extremes has been adequately modeled, $\mathrm{VaR}$ estimates for the daily returns distribution can be derived from estimates of lower quantiles of the extremes distribution using the expressions for the quantile functions found in Appendix A. VaR models can only be useful if their forecasts 
are sufficiently accurate and this is why any VaR model should be validated. Backtesting is the task of systematically comparing the VaR forecasts with the actual returns using historical data. The number of times that the VaR forecasts are violated by the actual returns can then be counted and this serves as an indication of how well calibrated a VaR model is. ${ }^{6}$ For example, a daily VaR model is assumed, which at the $c \%$ confidence level produces a total of $N$ violations over a testing period of $T$ days. If the model is accurate then the failure rate, $N / T$, should be an unbiased estimator of $p \%=1-c \%$, where $p$ is the probability of having a VaR violation. Formally, we want to know whether the number of violations is too large or too low at a given confidence level under the null hypothesis that $p \%=1-c \%$. This is particularly important since it is not desirable to either underestimate or overestimate risk. Kupiec (1995) proposed a test based on a likelihood ratio that balances the type 1 (rejecting a correct model) and type 2 (not rejecting an incorrect model) errors. This test is defined by:

$$
L R_{\mathrm{uc}}=-2 \ln \left[(1-p)^{T-N} p^{N}\right]+2 \ln \left\{\left[1-\left(\frac{N}{T}\right)\right]^{T-N}\left(\frac{N}{T}\right)^{N}\right\}
$$

this likelihood ratio test statistic is then asymptotically distributed as a chi-square with one degree of freedom.

However, this is a test that examines the average performance of a VaR model but says nothing about whether or not the violations occur in clusters (unconditional coverage (uc) test). Christoffersen (1998) extended this test to account for the serial independence of violations. This conditional coverage (cc) test uses an indicator that takes the value zero if the VaR estimate is not violated by the actual return and the value of one if it is. Then $\psi_{i j}$ can be defined as the number of returns in state $i$ while they have been in state $j$ previously and $\pi_{i}$ can be defined as the probability of having an exception that is conditional on state $i$ the previous day. Standard Markov chain theory can be used to show that the likelihood ratio test statistic that tests for independence in the series of total violations is given by:

$$
\begin{aligned}
L R_{\text {ind }}= & -2 \ln \left\lfloor(1-\pi)^{\left(\varphi_{00}+\varphi_{10}\right)} \pi^{\left(\varphi_{01}+\varphi_{11}\right)}\right\rfloor \\
& +2 \ln \left\lfloor\left(1-\pi_{0}\right)^{\varphi_{00}} \pi_{0}^{\varphi_{01}}\left(1-\pi_{1}\right)^{\varphi_{10}} \pi_{1}^{\varphi_{11}}\right\rfloor
\end{aligned}
$$

\footnotetext{
${ }^{6}$ This idea is central in the Basel Committee on Banking Supervision (1996) recommendations since in the absence of a validation method, financial institutions might have an incentive to underestimate market risk, thus assigning too little capital as an MCR. The basic idea is that if a model is perfectly specified then the number of reported violations over a time period should be in line with the confidence level. For example, at the $95 \%$ confidence level it is expected that the actual returns will be larger than the VaR forecasts only $5 \%$ of the time (eg, if 100 past daily returns are used, five VaR violations are expected).
} 
Combining this test with the test for unconditional coverage forms the test for conditional coverage:

$$
L R_{\mathrm{cc}}=L R_{\mathrm{uc}}+L R_{\mathrm{ind}}
$$

which is asymptotically chi-square distributed with two degrees of freedom.

Both tests are popular backtesting methods and, therefore, they are both employed to assess the accuracy of the VaR estimates generated by the models evaluated in this paper.

\section{DESCRIPTION OF THE DATA}

In order to assess the ability of the GEV and GL distributions to adequately fit extreme returns, data from three different markets were used. In particular, daily closing prices of the CAC-DS index for the French stock market ${ }^{7}$ and the Commodities Research Bureau (CRB) index for the commodities market were collected from Datastream over the period 1977 to 2006. For the bond market, however, daily prices for the German 10 year benchmark bond index were only available for the period 1980 to 2006 . This time span contains the rather volatile periods of 1978 to 1982,1986 to 1988,1990 to 1992 and 1997 to 2000 and 2001 to 2003 where some of the lowest daily returns for all three indexes occurred. In particular, some of the most important events contained in this 30 year period are the oil crisis in 1979, the global bond market crisis in the early 1980s, the collapse of the international stock markets in 1987, the Gulf crisis in 1991, the collapse of the exchange rate mechanism in 1992, the turbulence due to the Asian and the Russian financial crises in 1997 and 1998 respectively, and the terrorist attack on the US in September 2001.

Table 1 contains descriptive statistics of the three indexes daily returns. It can be noticed that the CAC-DS offered the highest mean daily return, 0.04\%; however, it was also the more risky with a daily standard deviation of $1.14 \%$. The lowest daily return was given by the CAC-DS index, $-9.89 \%$, and the highest by the German bond, $8.10 \%$. The values of skewness for the CAC-DS and CRB indexes indicate

\footnotetext{
${ }^{7}$ The index used in this paper is not the well known CAC40 index. Instead, the market-value weighted index that Datastream calculates is used and for familiarity reasons, it is denoted as CAC-DS. The use of the CAC-DS index offers a number of advantages compared with its CAC40 counterpart. Firstly, small capitalization firms are included and thus, the CAC-DS index can be regarded as a more vigorous representation of the French stock market. Secondly, any capital changes such as dividend distributions, rights offerings and stock splits are taken into account and therefore no additional adjustments have to be made. Finally, it is available for a relatively long period. This is extremely important for EVT analysis because the validity of asymptotic theory is heavily based on the size of the database available. For example, the CAC40 index started in 1987, while the CAC-DS index started in 1973. Thus, had we used the CAC40, half the data would be available for EVT analysis. The corresponding Datastream code is TOTMKFR and the index is composed of 250 of the most heavily traded shares that aim to cover the $70 \%$ to $80 \%$ of the total market capitalization. The CRB index is considered to be a benchmark for the commodities markets. It is made up of 22 basic commodities and is very sensitive to economic changes, thus indicating changes in business activity. Finally, the German 10 year benchmark bond is considered to be the barometer of the European bond markets.
} 
that the negative returns were larger than their positive counterparts while the reverse is true for the German bond. In addition, the kurtosis values for all indexes imply that the empirical distributions of daily returns were fat tailed. This finding was also confirmed by the Jarque-Bera normality test that rejected the normality assumption at the $5 \%$ significance level.

The daily returns of the three indexes can be further examined by standardizing them, computing the pairs of empirical percentiles $(1 \%, 99 \%)$ and $(5 \%, 95 \%)$ and comparing these with those of a standard normal distribution, ie, $(-2.326$, 2.326) and $(-1.644,1.644)$, respectively. For the CAC-DS, CRB and German bond indexes daily returns the pairs of the $(1 \%, 99 \%)$ and $(5 \%, 95 \%)$ empirical percentiles were found to be larger $((-2.884,2.607),(-2.588,2.533)$ and $(-2.586,2.893)$, respectively $)$, and smaller $((-1.563,1.487),(-1.679,1.594)$ and $(-1.548,1.612)$, respectively), respectively, confirming the presence of fat tails in the empirical distribution of the daily returns. Furthermore, under the normality assumption only 21 of 7,618 observations for the CAC-DS and CRB indexes and 18 of 6,847 observations for the German bond index would be expected to be outside the range plus or minus three standard deviations away from the mean; about 10 in each tail. However, for the CAC-DS, 103 observations were outside this range (64 in the left and 39 in the right tail), for the CRB, 75 observations were outside this range (40 in the left and 35 in the right tail) and for the German bond 102 were outside this range (40 in the left and 62 in the right). Hence, the hypothesis that the daily returns of the CAC-DS, CRB and German bond indexes follow a normal distribution can be rejected. In this case it is the extremes that mainly contribute to the non-normality of the daily returns distribution.

\section{ANALYSIS OF THE EXTREME RETURNS}

Weekly minima extremes were collected over the period 1977 to 2006 for the CAC-DS and CRB indexes and 1980 to 2006 for the German 10 year benchmark bond. Section 4.1 describes the identification of the appropriate distribution(s) and Section 4.2 details the estimation of parameters and the goodness-of-fit test.

\subsection{Identifying the distribution of the extreme minimum daily returns}

The L-skewness $\left(\tau_{3}\right)$ and L-kurtosis $\left(\tau_{4}\right)$ were calculated for the weekly minima of the three indexes and were plotted on an L-moment ratio diagram. Figures 1, 2 and 3 contain the $\tau_{3}$ and $\tau_{4}$ for the series of the weekly minima for the CAC-DS and CRB indexes divided into 30 subperiods and the German bond index divided into 27 subperiods, respectively. From an initial inspection of these figures, it seems that all the distributions can be excluded except for the GL and the GEV. This is because for all three indexes the points of the $\tau_{3}$ and $\tau_{4}$ are mainly dispersed 


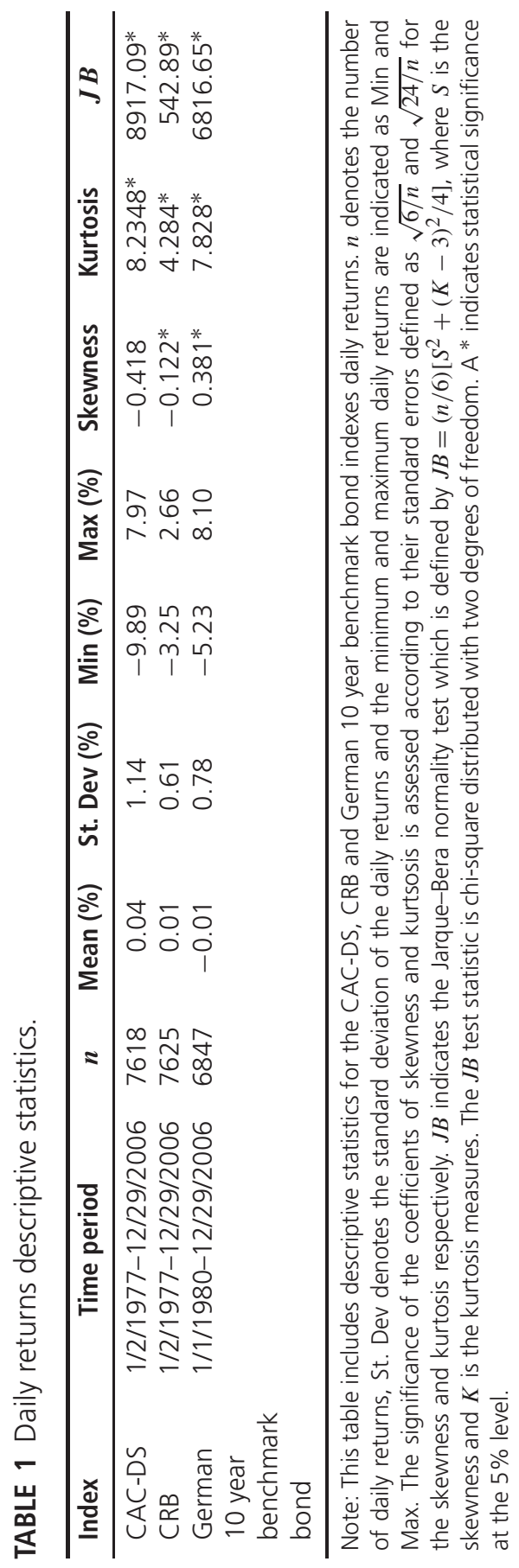


FIGURE 1 L-moment ratios diagram for the CAC-DS weekly minima.

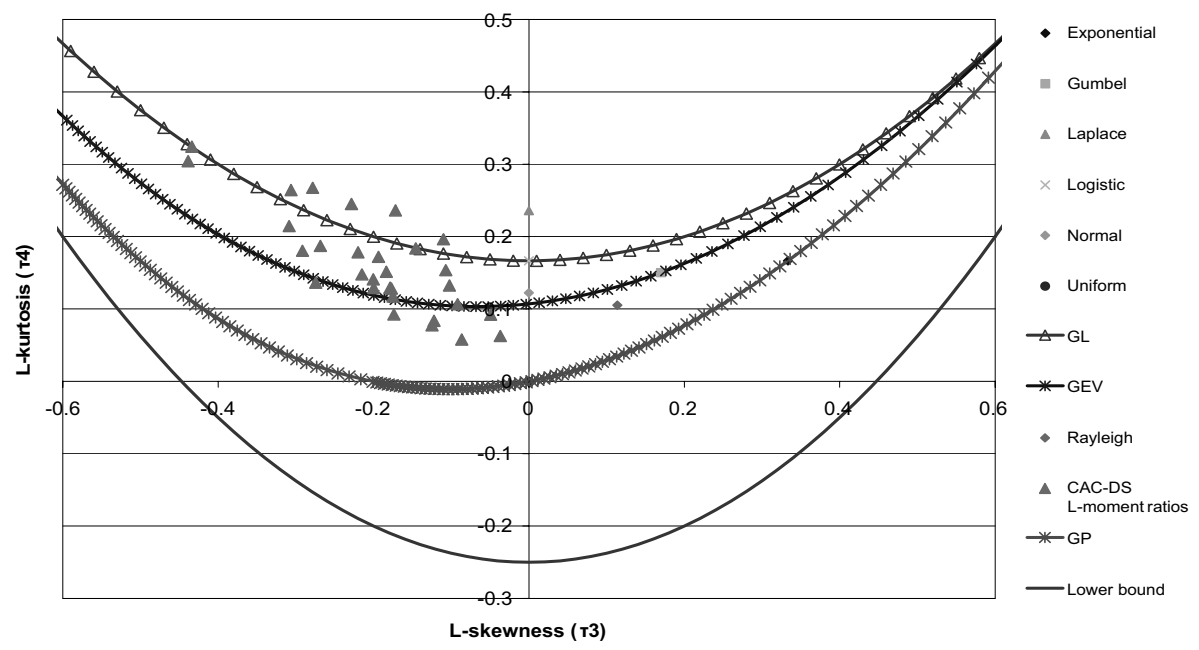

Note: This diagram illustrates the L-moment ratios points for the CAC-DS index daily returns weekly minima, divided into 30 subperiods, over the period 1977 to 2006. The plots of the L-skewness and L-kurtosis are mainly concentrated around the theoretical curves of the GL and the GEV distributions indicating that these two distributions are likely to adequately fit the empirical data.

around the theoretical curves of the GL and the GEV distributions. ${ }^{8}$ However, in order to choose between the GL and the GEV distribution, further analysis is required and a more formal test of goodness-of-fit of these two distributions should be applied.

\subsection{Parameter estimates and goodness-of-fit test}

The GL and GEV distributions were fitted to the weekly minima for the whole interval and for the different subdivisions of the extremes for all three indexes with the parameters being estimated by the PWM method. The parameter estimates together with their standard errors and the $p$-values of the Anderson-Darling (AD) goodness-of-fit test for the CAC-DS, CRB and German bond indexes are contained in Tables 2, 3 and 4 respectively. When the weekly minima of all indexes for the whole interval were fitted by either the GL or GEV distributions, the AD goodness-of-fit test indicated that both distributions provide a marginally adequate fit to the CAC-DS empirical data, with $\mathrm{AD} p$-values higher than 0.05 but lower than 0.100 , and an inadequate fit to the CRB and German bond indexes

\footnotetext{
${ }^{8}$ The corresponding L-moment plots were also generated for the 10 subdivisions of the weekly minima and similar patterns appeared. In the interest of brevity these diagrams are not included in the paper; however, they are available from the author upon request.
} 
FIGURE 2 L-moment ratios diagram for the CRB index weekly minima.

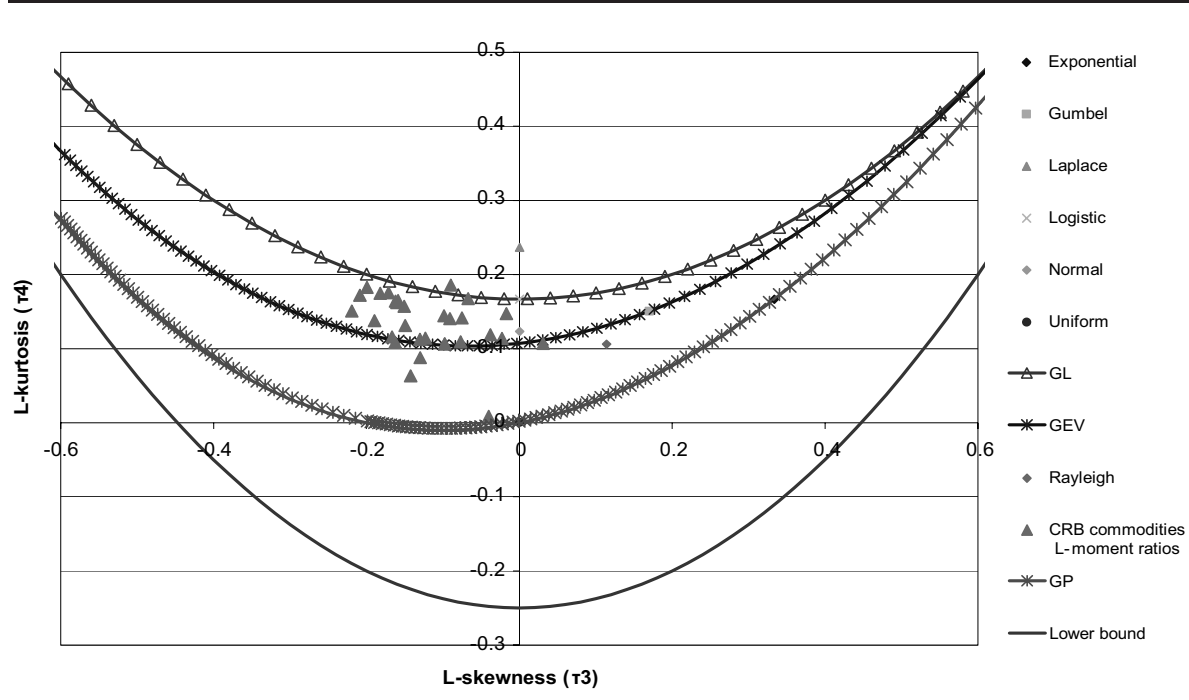

Note: This diagram illustrates the L-moment ratios points for the CRB index daily returns weekly minima, divided into 30 subperiods, over the period 1977 to 2006 . The plots of the L-skewness and L-kurtosis are mainly concentrated around the theoretical curves of the GL and the GEV distributions indicating that these two distributions are likely to adequately fit the empirical data.

FIGURE 3 L-moment ratios diagram for the German 10 year benchmark bond weekly minima.

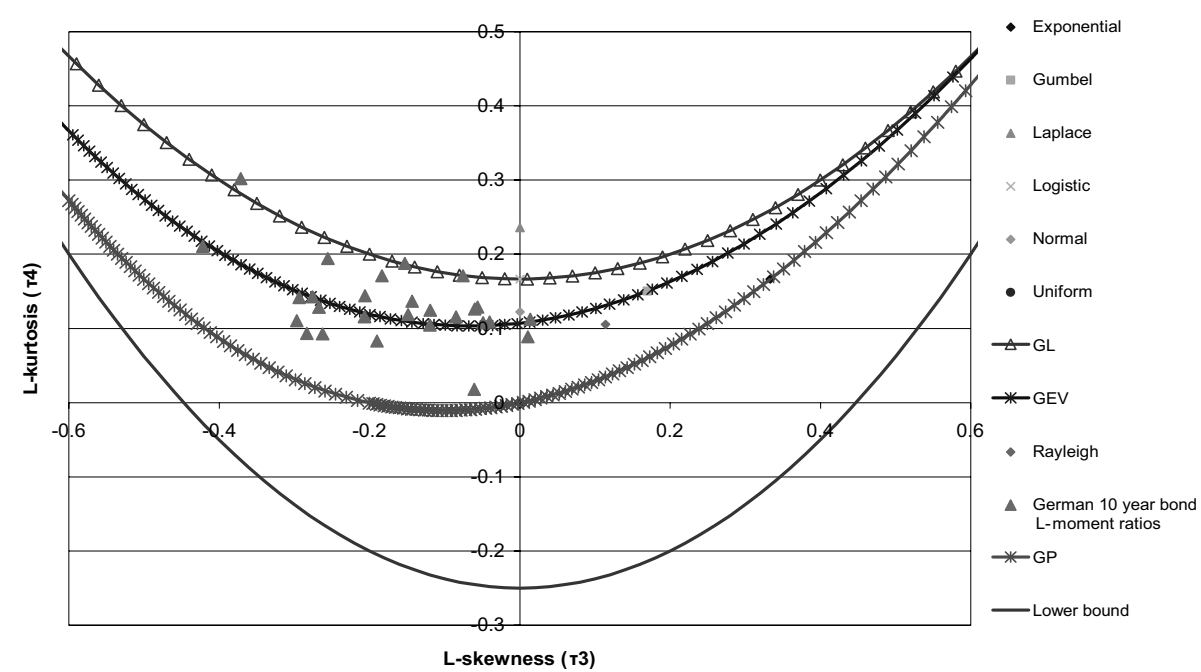

Note: This diagram illustrates the L-moment ratios points for the German 10 year benchmark bond daily returns weekly minima, divided into 27 subperiods, over the period 1980 to 2006. The plots of the L-skewness and L-kurtosis are mainly concentrated around the theoretical curves of the GL and the GEV distributions indicating that these distributions are likely to adequately fit the empirical data. 


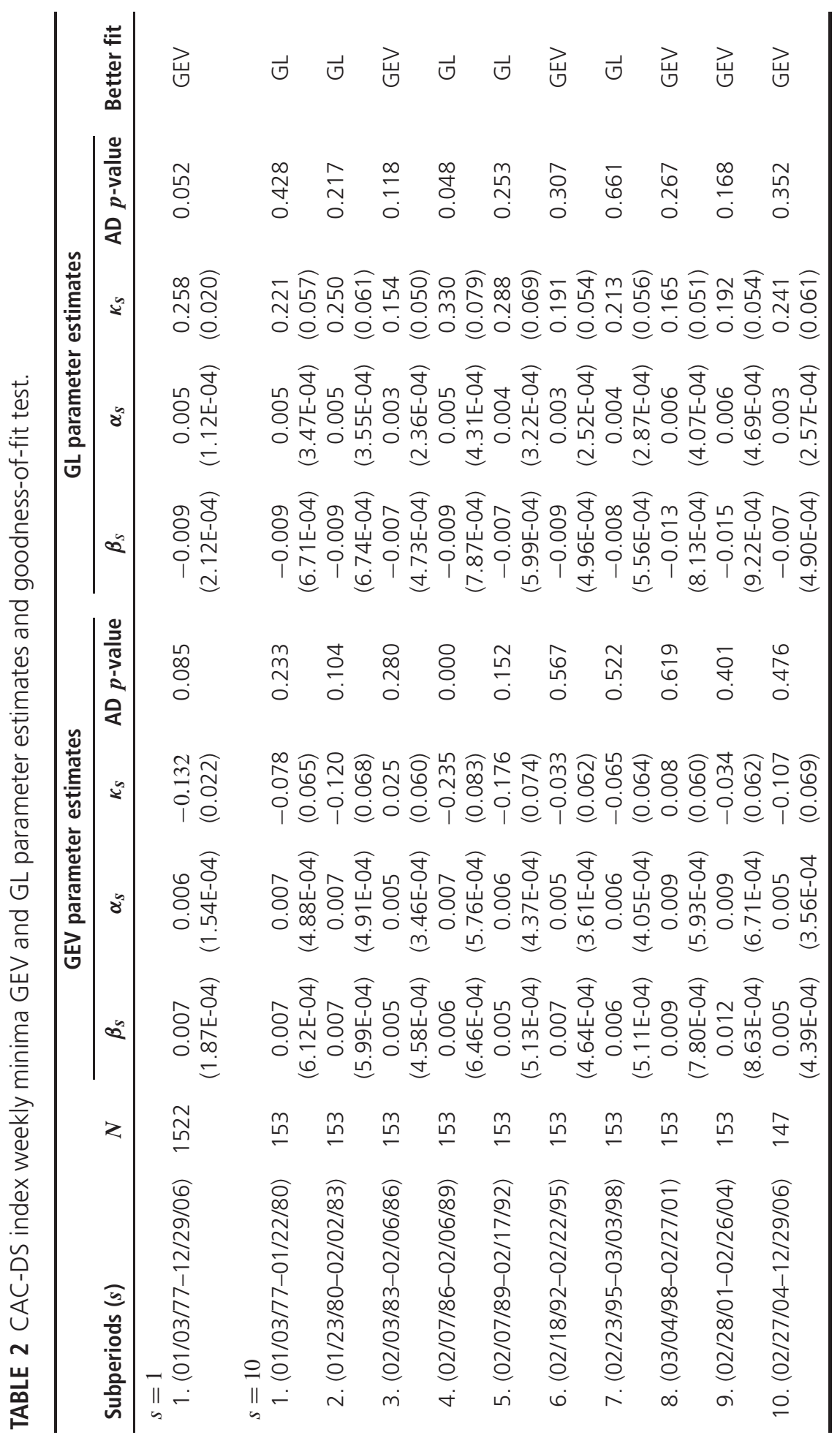




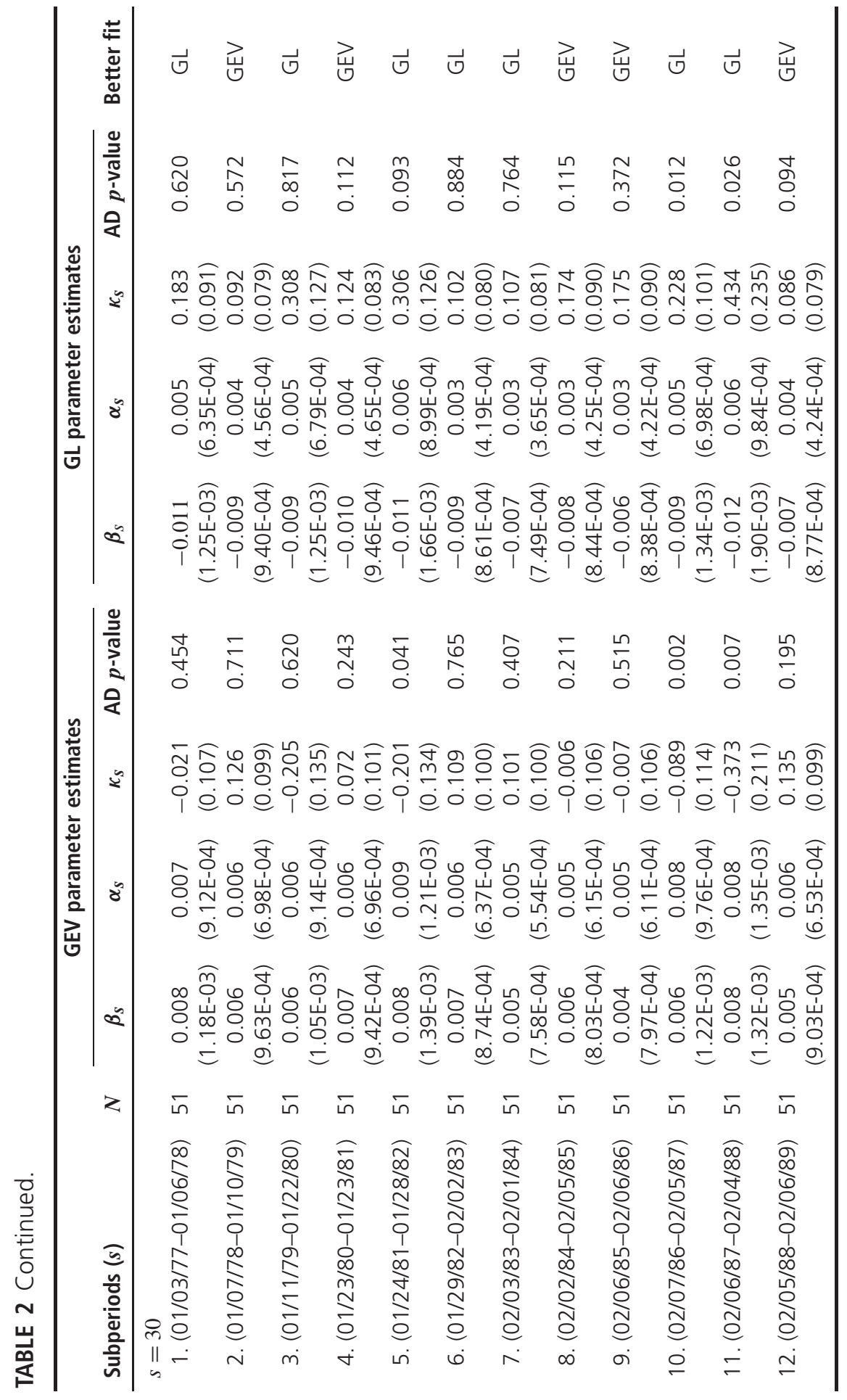




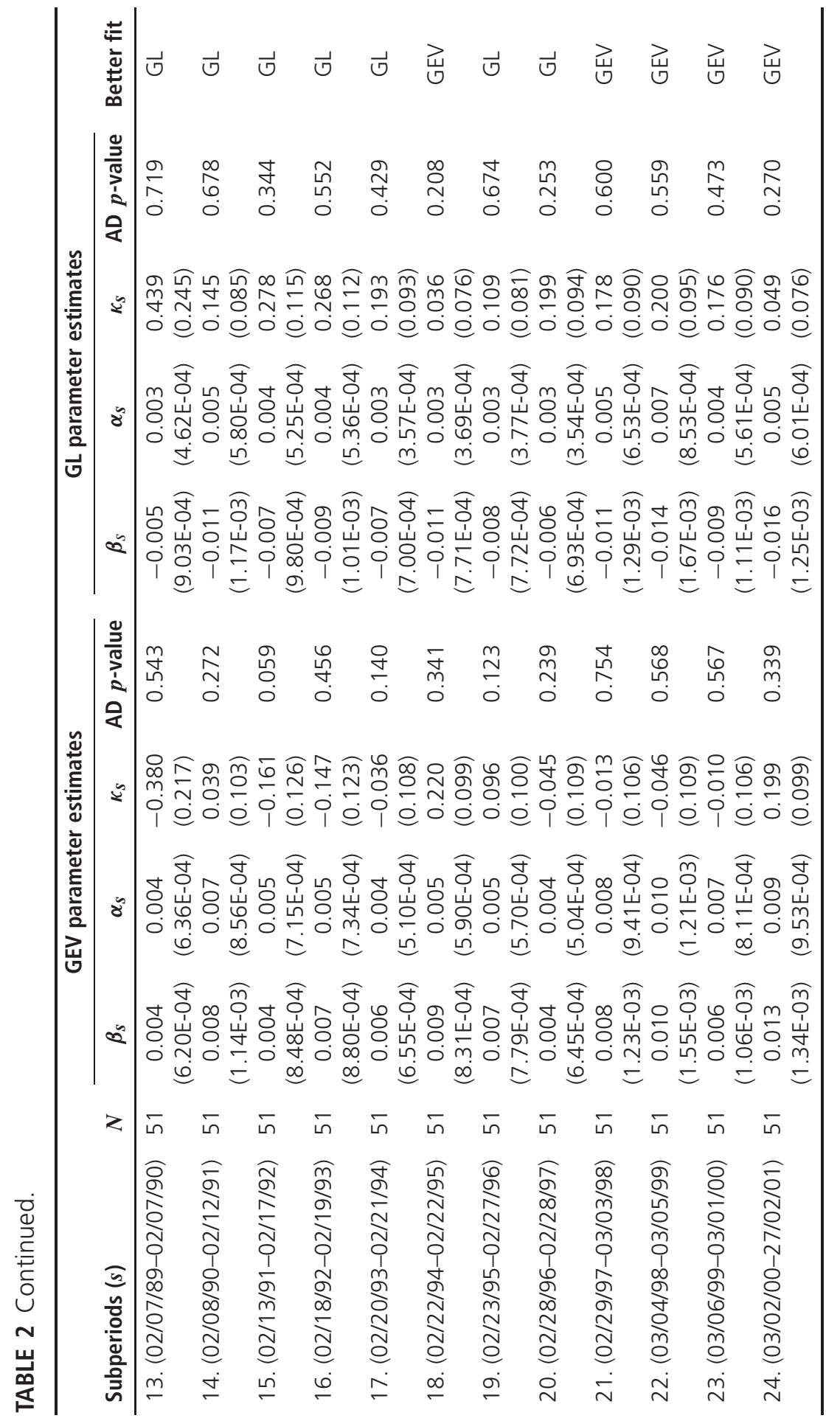




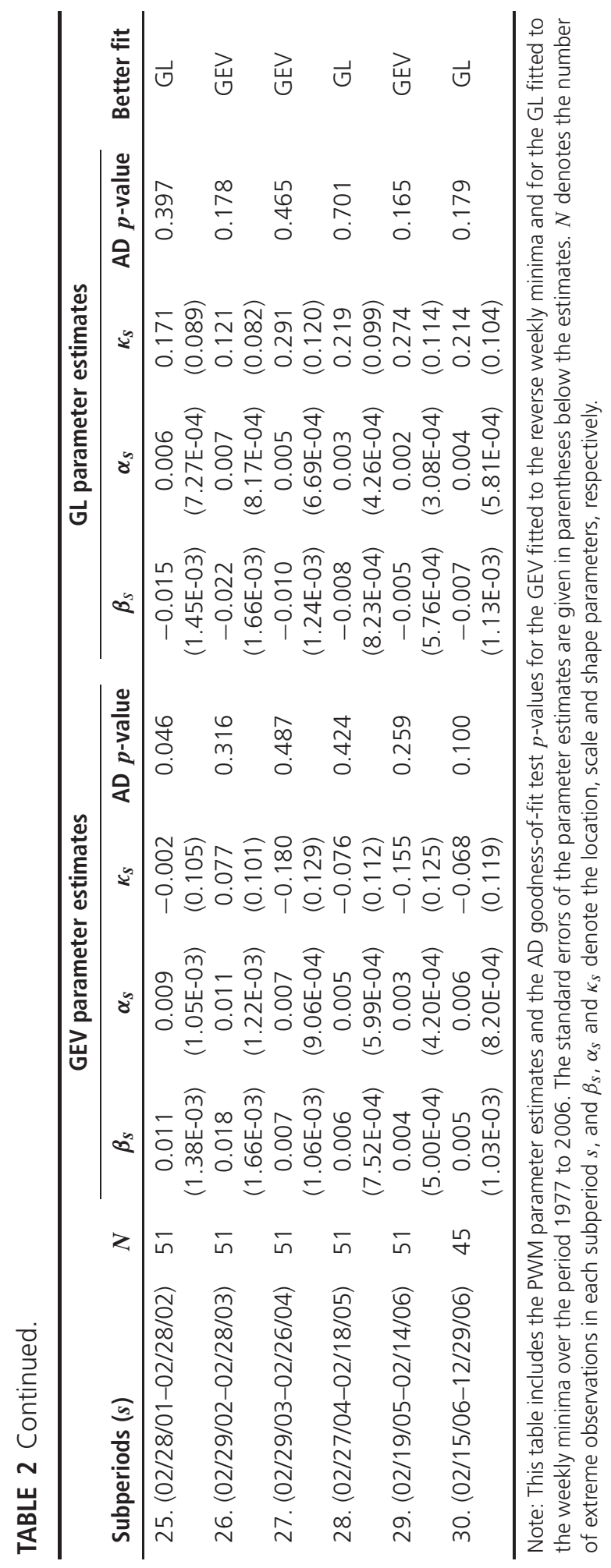


with $\mathrm{AD} p$-values lower than 0.05 . One possible explanation is that the nature of the distribution of the extremes was changing over time and, therefore, when long time-periods were used the data came from a mixture of distributions; thus, it was difficult for a single distribution to provide a superior fit.

However, when the weekly extremes were divided into 10 subperiods, the fit that the two candidate distributions provided considerably improved. For example, in the case of the CAC-DS index, both candidate distributions provided an adequate fit in nine of the 10 subperiods while in comparison to each other, both fitted better than the other in the same number of subperiods, ie, in five (Table 2). It can be noticed, however, that both the GEV and GL distributions failed to adequately fit the extremes in subperiod four which corresponds to a period which contains the stock market crash in October 1987. It can also be noticed that in this subperiod the shape parameter takes its maximum value, in absolute terms, for both distributions indicating a fat-tailed distribution. Similarly, the fitting that the GEV and GL provided to the weekly extremes of the CRB index considerably improved when the data was divided into 10 subperiods (Table 3). Both distributions fitted adequately in nine of the 10 subperiods while in comparison each fitted better than the other one in five subperiods. It can be noticed that both the GEV and GL failed to fit the extreme data in subperiod nine, which is associated with the terrorist attack on the US in 2001, and the negative market sentiment that followed. Finally, when the weekly extremes of the German bond index were divided into 10 subperiods, the GL managed to fit better than the GEV in six of these (Table 4). In addition, the GL fitted adequately in eight subperiods while the GEV fitted adequately in seven.

When the weekly extremes were divided into 30 subperiods, both the GEV and GL distributions appeared, in general, to be capable of describing well the behavior of extremes of the CAC-DS, CRB and German bond indexes. In particular, in the case of the CAC-DS, the GL distribution fitted adequately in 28 of the 30 subperiods, with $\mathrm{AD} p$-values within the range 0.012 to 0.884 , while the GEV fitted in 26, with AD $p$-values ranging from 0.002 to 0.765 (Table 2). In comparison to the GEV, the GL fitted better in 17 of the 30 subperiods. In addition, the effects of domestic and international events become apparent in subperiod five that is associated with the stock market turbulence due to the nationalization of many large French companies, and periods 10 and 11 that are associated with major political changes and the stock market crash respectively. Unsurprisingly the $p$-values of the AD goodness-of-fit test were low: $0.041,0.002$ and 0.007 for the GEV and 0.093, 0.012 and 0.026 for the GL for subperiods 5, 10 and 11 respectively. One can notice, however, that both the GEV and GL distributions can adequately fit the extreme daily returns in a number of other volatile time periods; for example, the 1991 Gulf war (period 15), the European exchange rate mechanism crisis in 1992 (period 16), the Asian crisis in 1997 (period 21), the global turmoil due to the demise of the Long Term Capital Management hedge fund (22) and the terrorist attack on the US in 2001 (period 25).

The observations in the case of the CRB and the German bond indexes are similar. In short, in the case of the CRB index, the GL provided a better fit in 


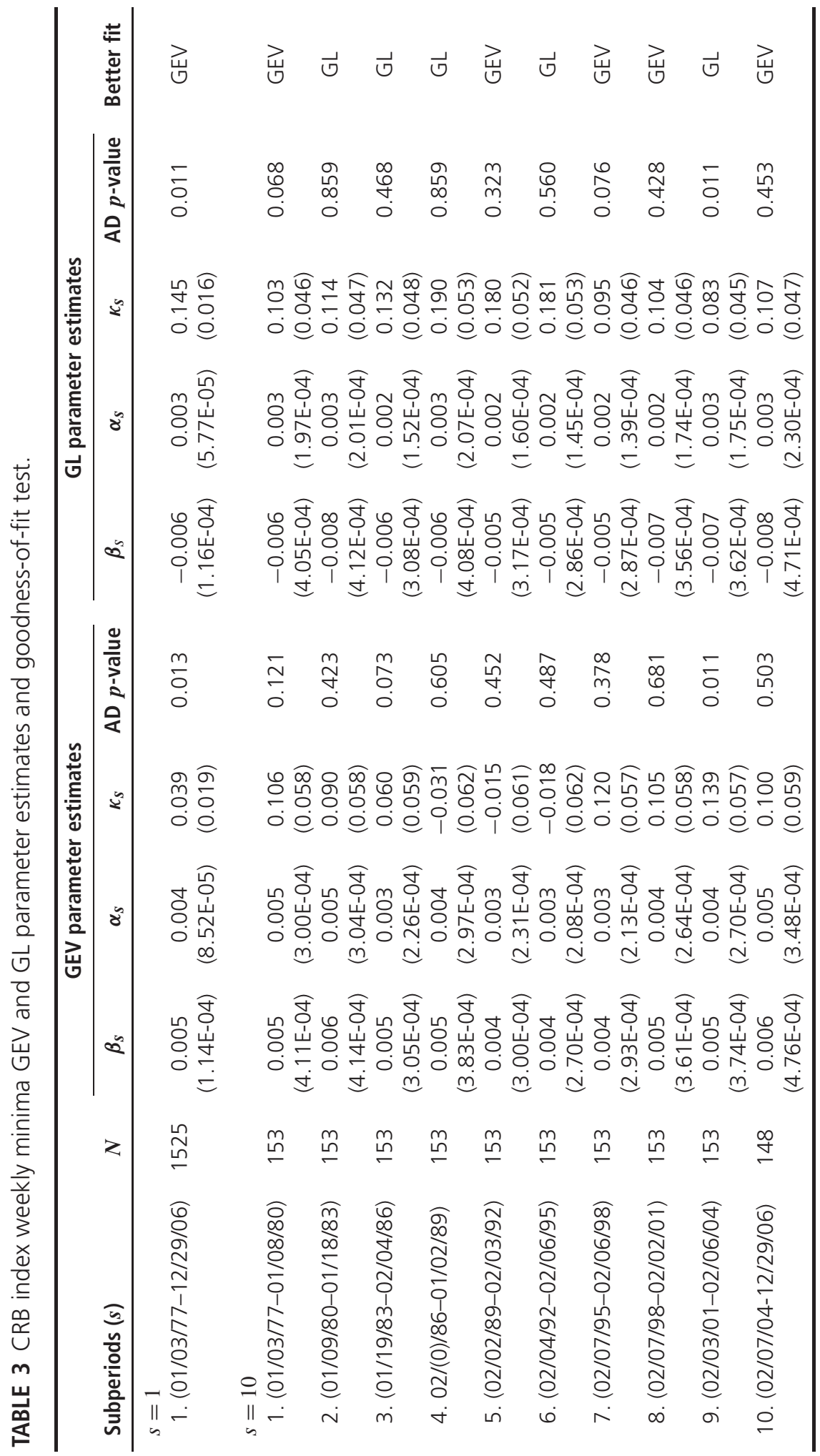




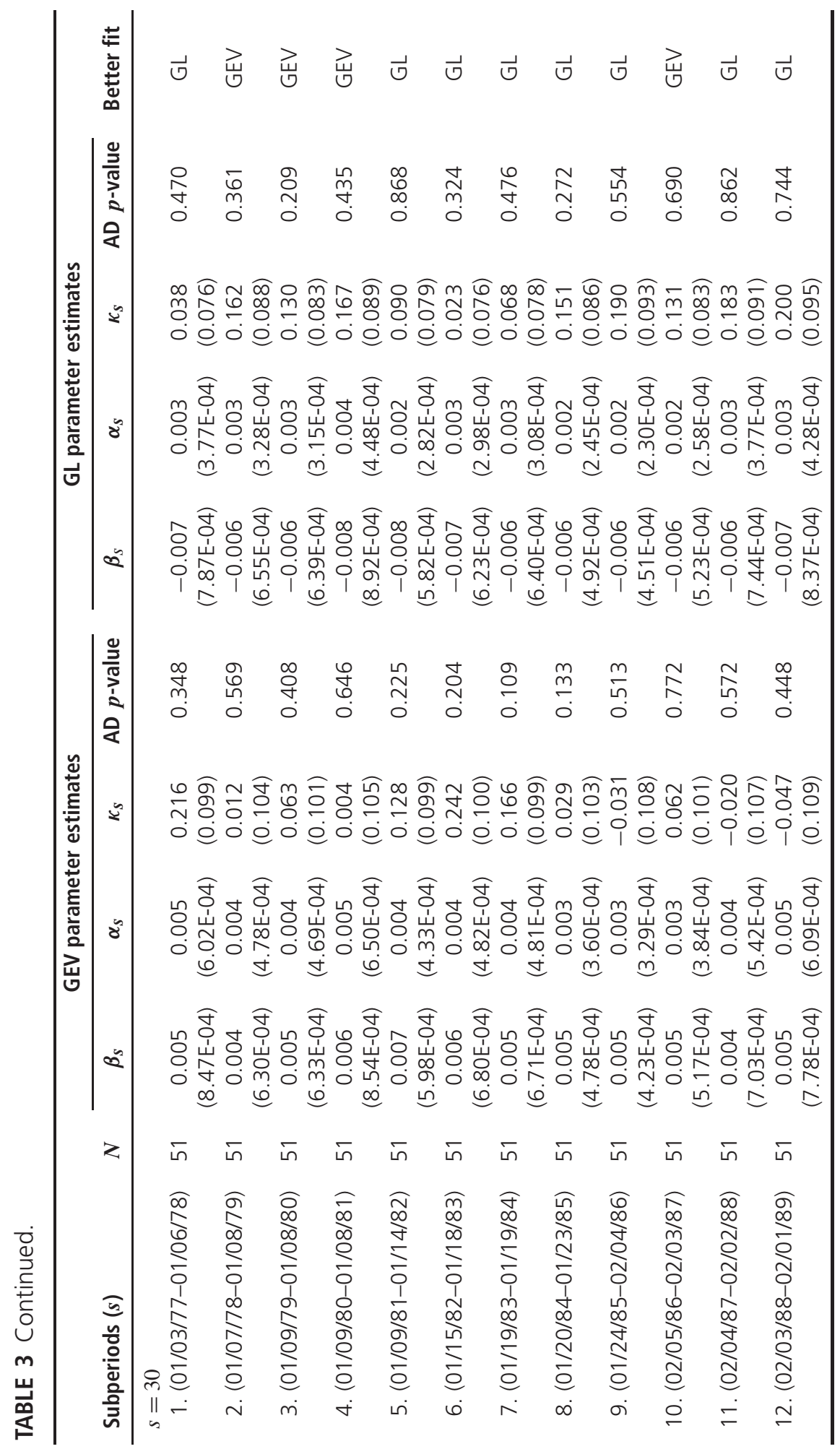




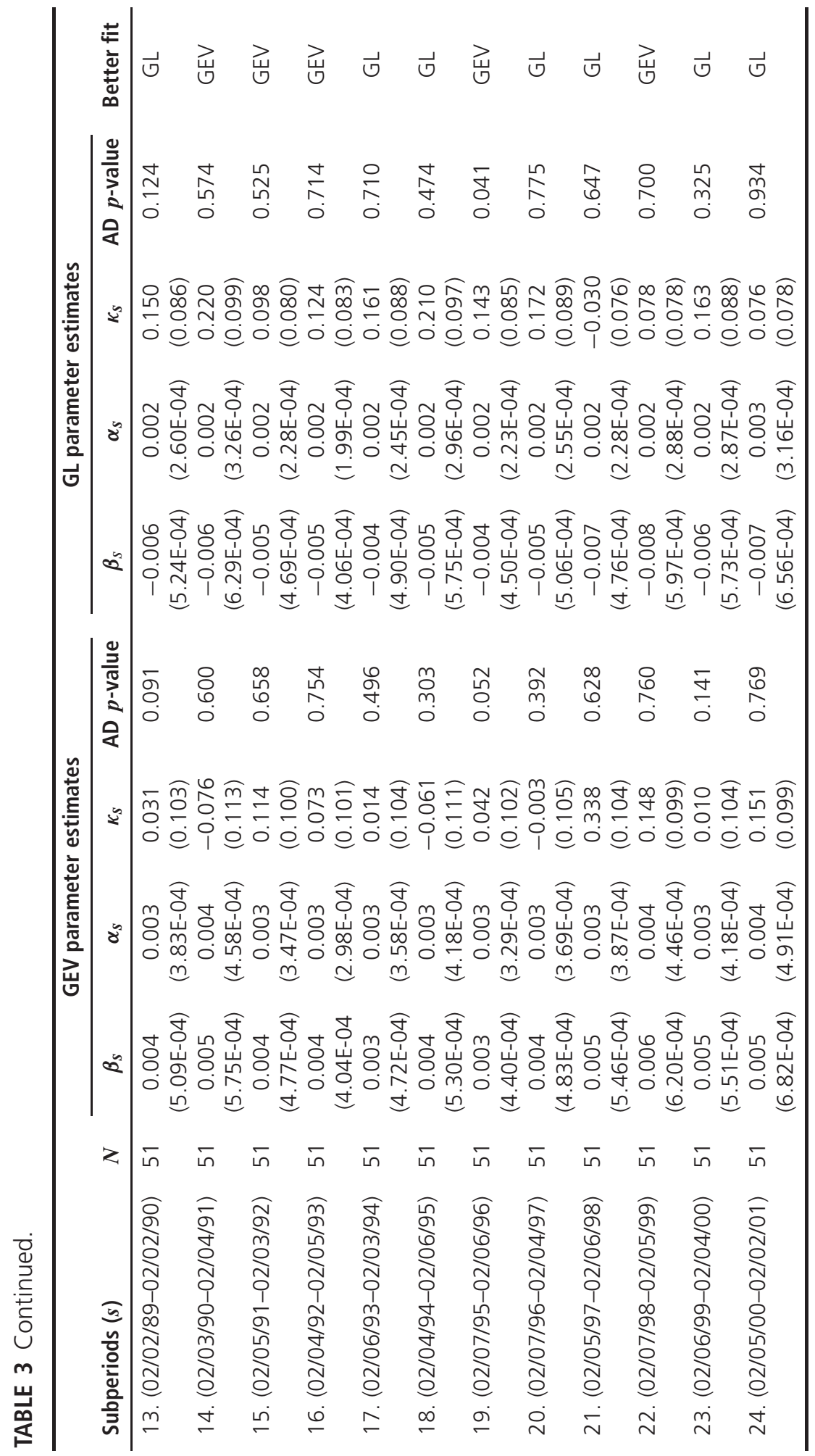




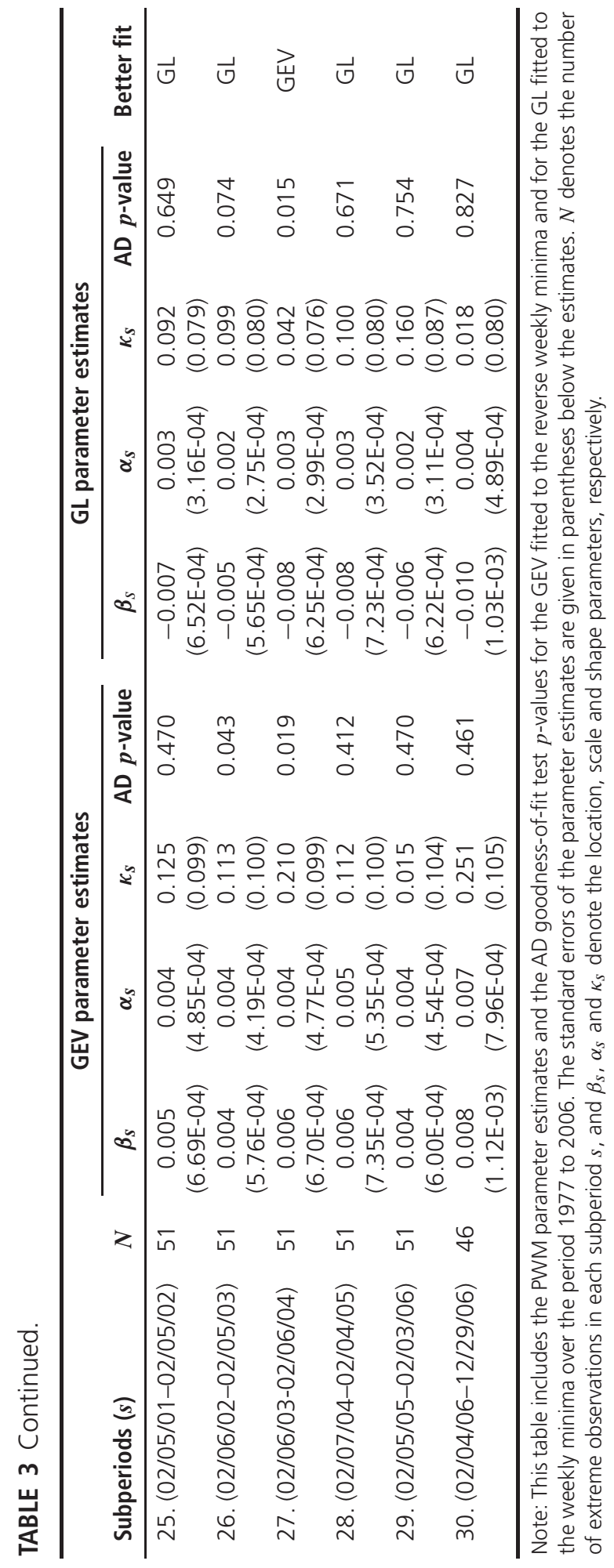


20 subperiods while the GEV provided a better fit in only 10 of the 30 subperiods (Table 3). However, both provided an adequate fit in 28 of the 30 subperiods. The $p$-values of the AD test were rather low in subperiods 26 and 27 and this can probably be attributed to the volatile markets after the terrorist attack on the US in September 2001. In the case of the German bond index weekly extremes divided into 27 subperiods, the GL adequately described the empirical data in 20 subperiods while the GEV only in seven of the 27 subperiods (Table 4). Although both candidate distributions provided an adequate fit in 20 subperiods with AD $p$ values higher than 0.05 , they did not manage to fit well the extremes in subperiods $1,2,4,5,12,13$ and 19. Subperiods 1 and 2 contain the bond market crash in the early 1980s due to the inflationary fears and the Soviet invasion of Afghanistan which followed a speculative boom in bonds in the late 1970s. In 1982 the bond market started to recover but in the period 1983-1984 (subperiods 4 and 5) it declined again, mainly due to inflationary expectations. Subperiods 12 and 13 correspond to the period 1991-1993 during which the global markets were rather volatile because of the Gulf war and the collapse of the exchange rate mechanism.

In summary, both the GEV and GL distributions appeared to be able to adequately model the extreme minima of the CAC-DS, CRB and German bond indexes daily returns over the periods studied. However, overall the GL distribution provided a better fit than the GEV when several subperiods were used. This is illustrated in Figure 4, which contains the lower tail cdfs of the empirical, normal, GEV and GL distributions, where the superior fitting that the GL provides to the really ruinous events located deep into the lower tail of the distribution becomes apparent.

This is an important result because current applications of EVT in finance focus on either the GEV or GP distributions and since these are less fat tailed than the GL there is considerable chance that the probabilities of extreme events are underestimated. Additionally, it seems that the nature of extremes changes over time since the behavior of the shape parameter for both distributions varied substantially across different subperiods. In particular, volatile subperiods that contained large negative daily returns tended to result in higher shape values than periods of low volatility that contained fewer and smaller negative daily returns. This is expected to have a significant effect upon VaR estimates and one would expect VaR estimates to be higher when the shape parameter values were higher. Such a result would naturally lead to larger MCR for banks if they were to be protected against large negative price movements. It seems that parameter estimates that correspond to short subperiods should be used in VaR estimation since this would allow VaR to respond quickly to the changes of the macro- and microeconomic conditions prevailing in the market place. It can also be noticed that the sign of the shape values for the GEV distribution changes over time indicating that there is no unique distribution within the GEV family of distributions that describes the empirical data well. This is in disagreement with Gettinby et al (2004) and Longin (1996), who detected no sign of changes when they fitted the GEV distribution to the extreme returns of the UK and US stock markets, respectively. Finally, one could also observe that the parameter estimates 


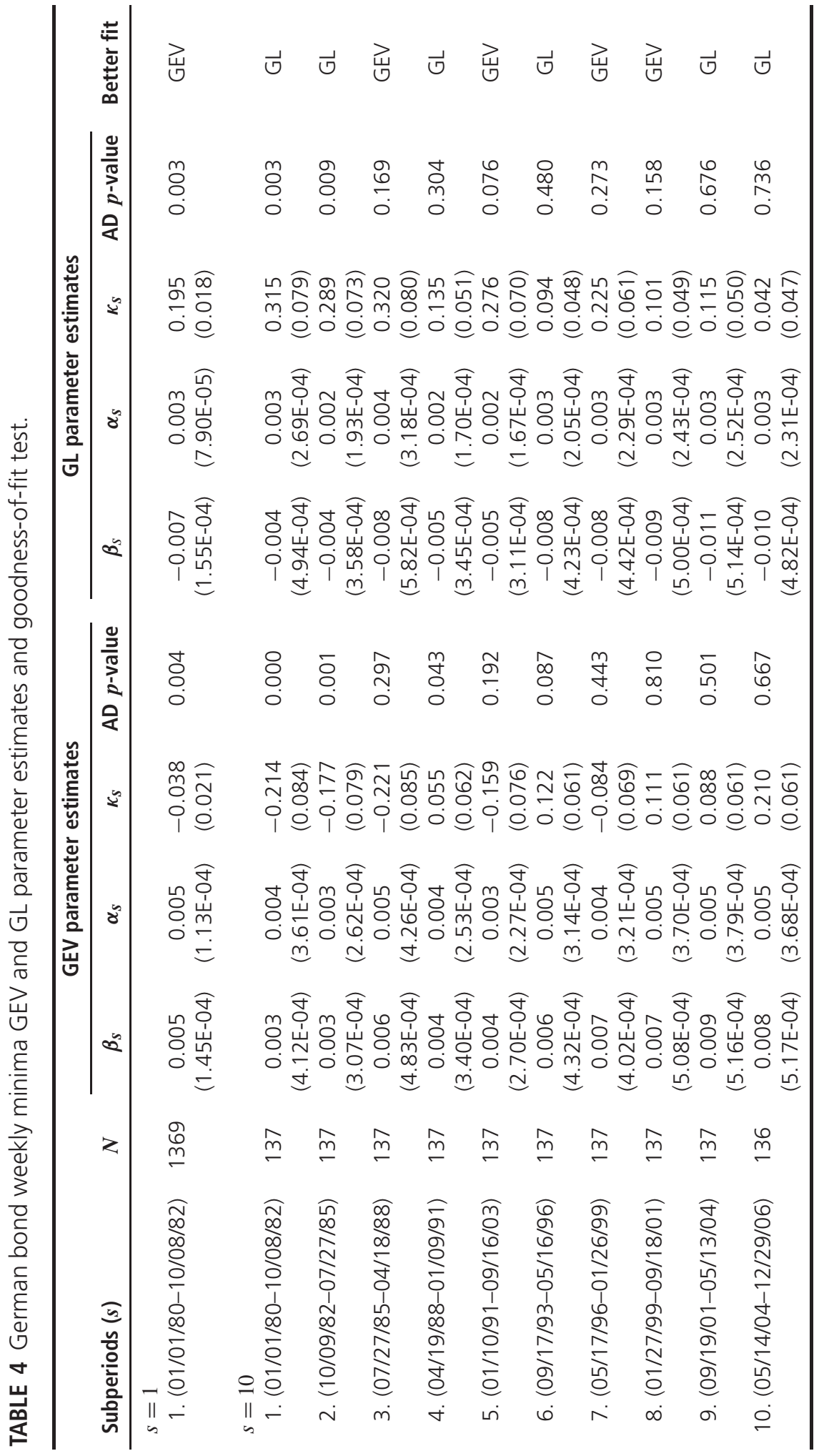




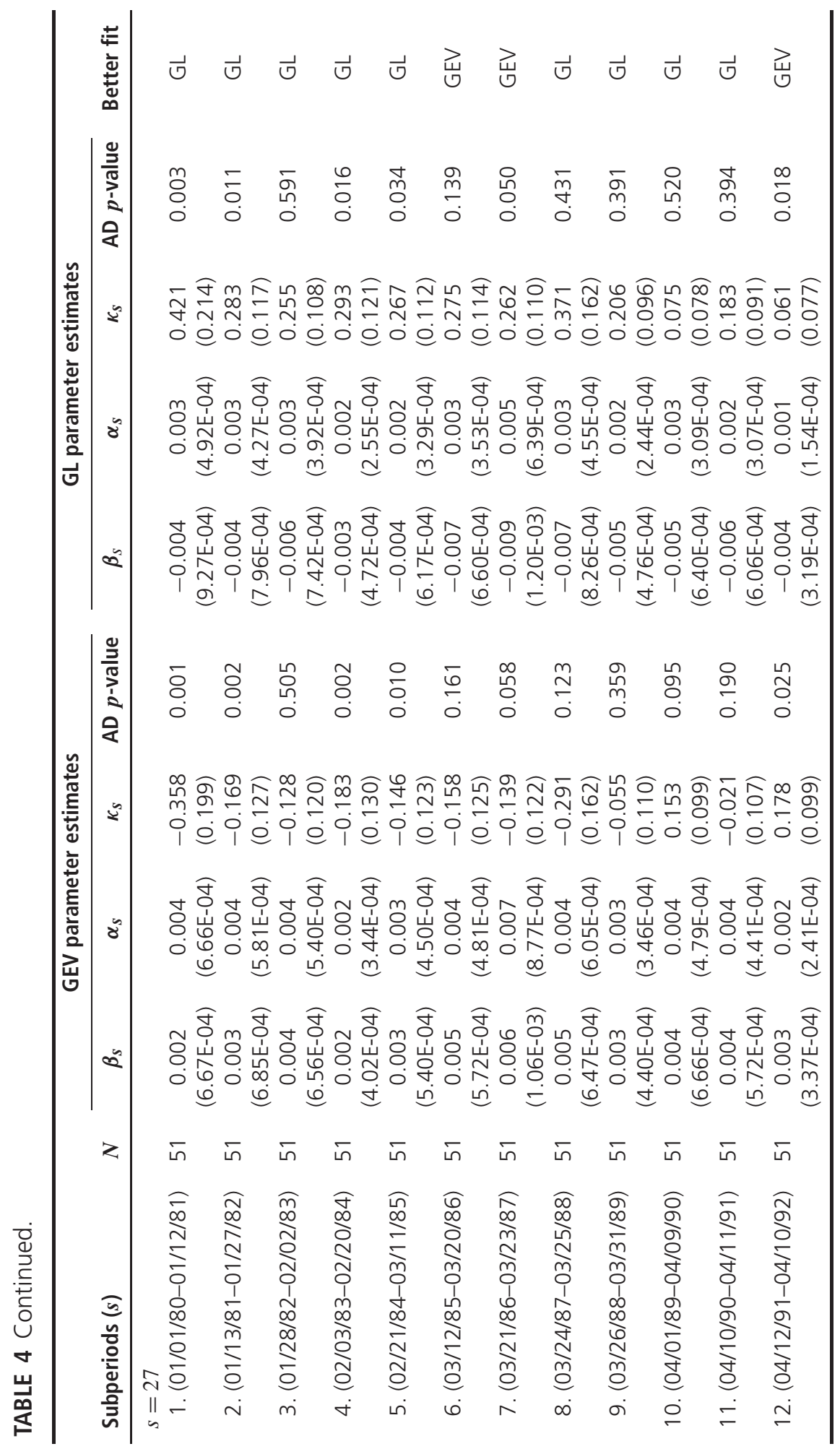




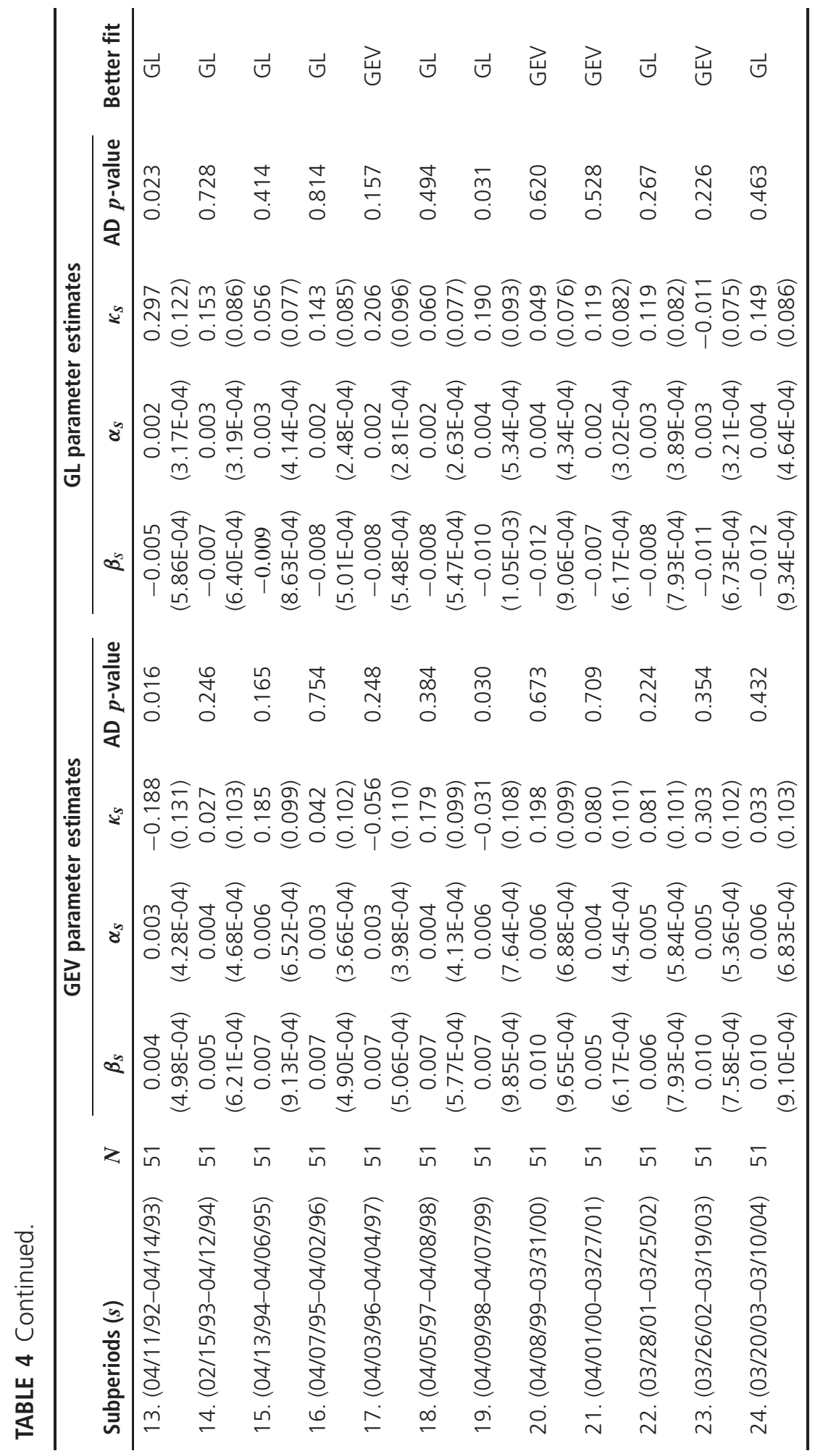




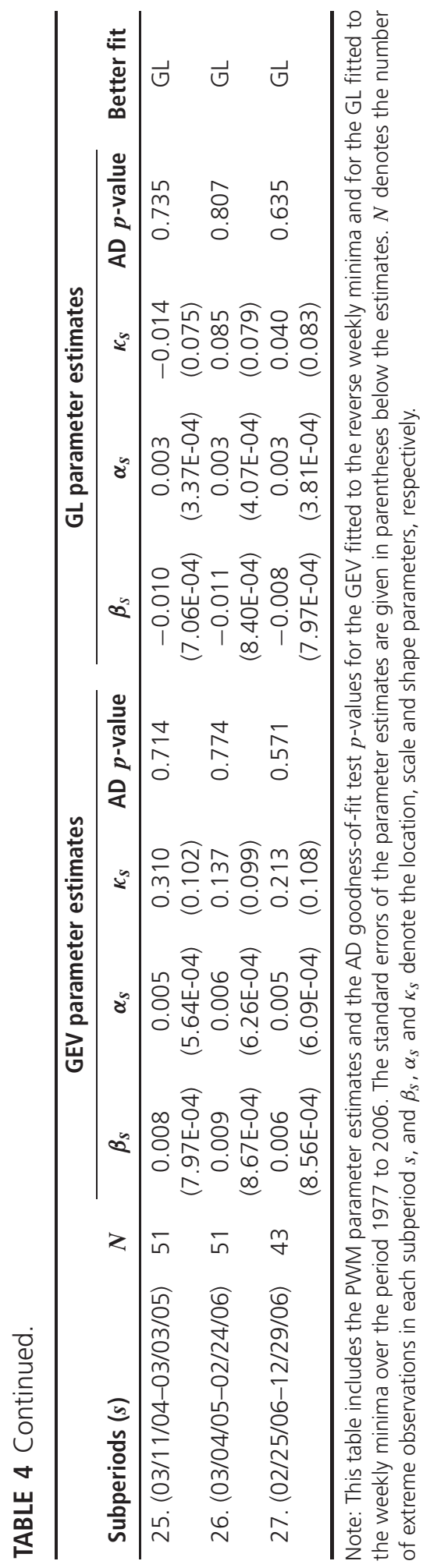


FIGURE 4 CAC-DS daily returns lower tail fitted by the normal, GEV and GL distributions.

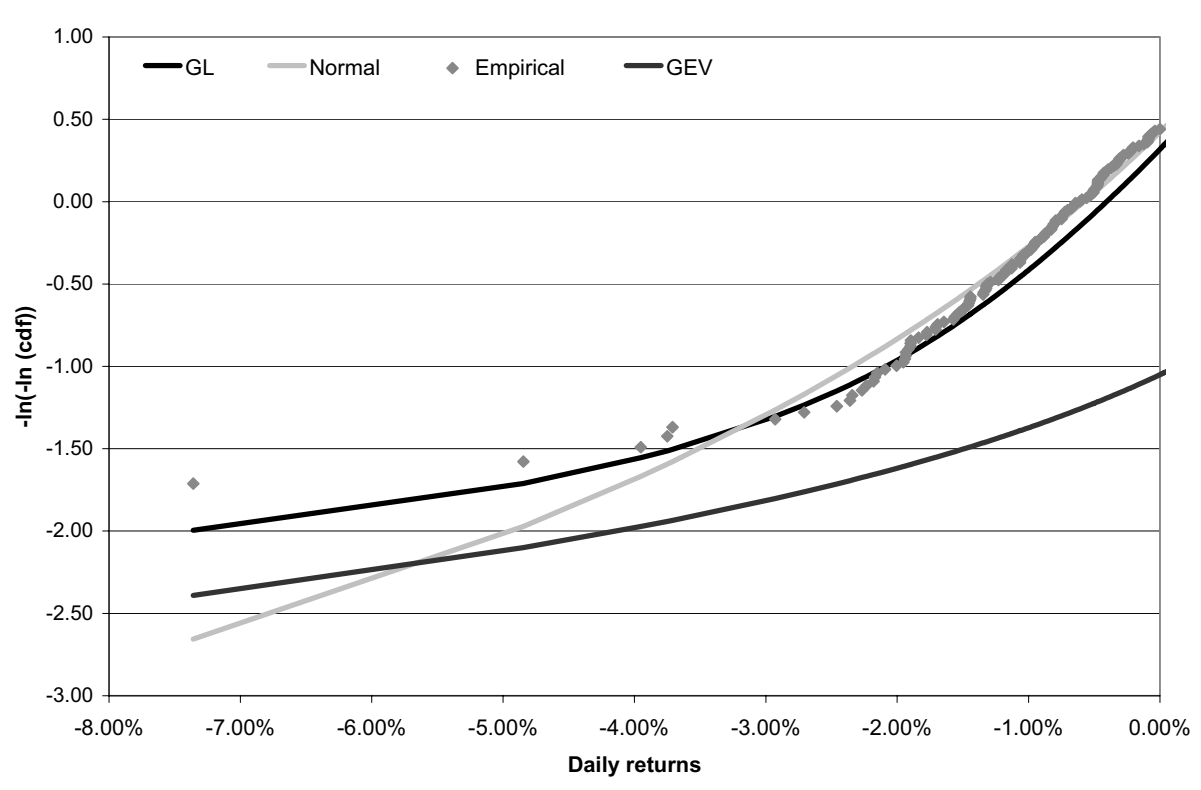

Note: This diagram illustrates the empirical distribution function (in log scale) of the CAC-DS index daily returns and the fit that the cdfs of the normal, GEV and GL distributions provide over the period 02/28/0 to $02 / 28 / 02$.

standard errors are low for the location and scale but rather large for the shape parameter; in general, the larger the dataset fitted the smaller the standard errors that can be attributed to the larger number of observations.

\section{ESTIMATING VALUE-AT-RISK USING EXTREME VALUE THEORY}

VaR estimates for the CAC-DS, CRB and German bond indexes daily returns were derived from lower quantiles of the distribution of extremes using the parameter estimates associated with the GEV and GL distributions. For the CAC-DS and $\mathrm{CRB}$ indexes the 30 subdivisions and for the German bond the 27 subdivisions of the weekly extremes time series were chosen to allow the parameters to change relatively frequently; about every year (static approach). However, the indication that the nature of extremes distribution is time variant suggests that more frequent updating of parameters might be more realistic. Consequently the parameters for the weekly minima were also estimated using moving windows of lengths 50, 
100, 200 and 300 (moving window approach). ${ }^{9,10}$ The set of confidence levels used comprise $97.50 \%, 99.00 \%, 99.50 \%, 99.75 \%$ and $99.90 \%$. For comparison, VaR estimates generated by traditional methods such as VC, HS, EWMA and Monte Carlo simulation based on the normal distribution were also derived. For these methods $250,500,1,000$ and 1,500 past daily returns were used for model calibration. ${ }^{11}$ In order to examine the performance of each approach the results were out-of-sample backtested over the period January 2, 1987 to December 31, 1991; this period contains some of the largest negative daily returns in all three markets. In addition, this period is of large enough size $(1,267,1,275$ and 1,260 daily returns for the CAC-DS, CRB and German bond indexes, respectively), to be considered adequate for statistical evaluation. Tables 5, 6 and 7 contain the VaR backtesting results for the CAC-DS, CRB and German bond indexes, respectively, which are presented in terms of the number of VaR forecasts violations by the actual returns followed by the corresponding Kupiec and Christoffersen test statistic $p$-values.

For the CAC-DS index the time period January 2, 1987 to January 2, 1992 is a rather volatile one (standard deviation is $1.18 \%$ ) with negative skewness $(-1.109)$ and high kurtosis (12.893). Unsurprisingly, the VC method underestimated risk by a considerable amount since it gave more violations than would be expected from an accurately calibrated model; however, the underestimation was not very severe at the $97.50 \%$ confidence level but it became worse as we moved further into the lower tail. The inability of this model to adequately describe the tails of the returns distribution is rather serious; for example, at the $99.90 \%$ confidence level only

\footnotetext{
${ }^{9}$ The underlying principle behind the choice of the number of daily returns used for the traditional methods, the number of subperiods into which the minima were divided in the static approach and the length of the moving window used, was to compare the VaR results based on the same informational time periods. For example, 250 daily returns correspond to about one trading year. When the series of weekly minima is divided into 30 subperiods the parameters derived also correspond to about one year. The same is true when a moving window of length 50 weekly minima is used.

${ }^{10}$ Gençay and Selçuk (2004) argued that the usefulness of EVT methods in VaR estimation can be enhanced by allowing for the possibility that the parameters may change over time. There have been attempts to take into account the time varying distributional characteristics of the extremes by using autoregressive processes (McNeil and Frey (2000); Pownall and Koedijk (1999)) or quantile regression techniques (Engle and Manganelli (2004)). More recently, Bali and Weinbaum (2005) introduced a conditional EVT volatility estimate, while Bali and Neftci (2003) modeled the parameters of the extremes' distribution as a function of past information in order to capture the time varying nature of extremes. However, these approaches introduce yet more parameters in to the modeling procedure and this is likely to result in larger estimation errors and possibly even more inaccurate VaR estimates.

${ }^{11}$ In order for the EWMA method to effectively take account of 250, 500, 1,000 and 1,500 past daily returns in the estimation of volatility, the parameter $\lambda$ was set to $0.996,0.998,0.999$ and 0.9993333332, respectively. For the Monte Carlo simulation method the normal distribution was assumed and for each daily VaR estimate 10,000 random scenarios were generated. Use of fewer than 250 and 500 past daily returns for the historical simulation method makes it impossible to generate estimates at some of the highest confidence levels because the dataset becomes too small. For example, the calculation of $99.90 \%$ VaR requires at least 1,000 daily returns.
} 


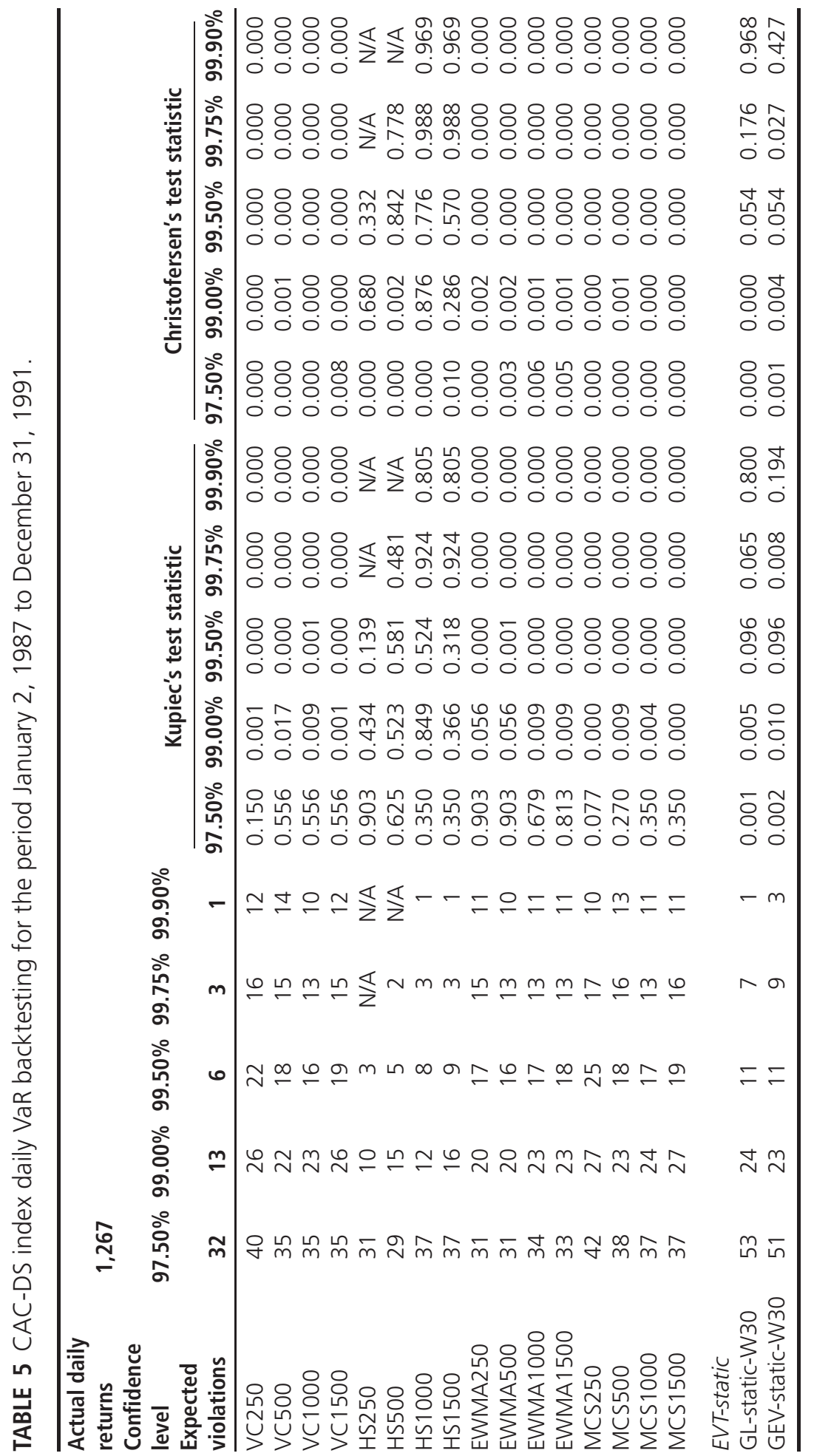




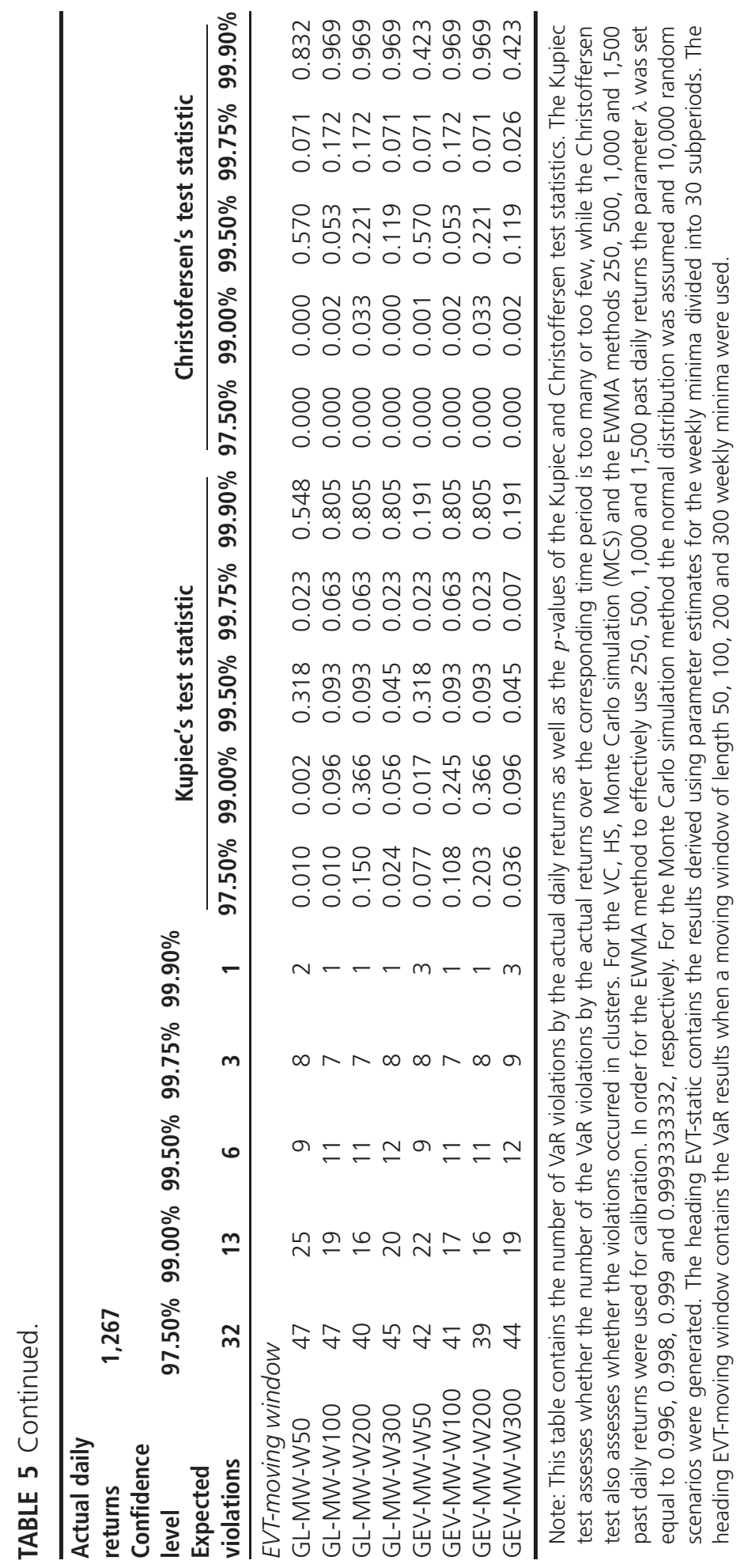


one violation was expected but the VC method provided 10 to 14 violations. ${ }^{12}$ The HS method, on the other hand, provided much better results, especially at high confidence levels where the $p$-values of both the Kupiec and Christoffersen test statistics were acceptable. As the number of past daily returns used increased, the HS provided very good results at the higher confidence levels of $99.75 \%$ and $99.90 \%$, although its accuracy decreased at lower confidence levels. Finally, the EWMA and MCS methods were the least accurate models at all confidence levels, with the exception of the EWMA method at the $97.50 \%$ confidence level. This was probably because the EWMA tends to react quickly to volatility changes but only after the event, while for the MCS it is probable that the normal distribution was not a good model for the daily returns over this time period.

The VaR results generated using EVT methods were examined next, starting with the static approach. It is noticeable that both the GEV and GL distributions underestimated risk with more violations being recorded than would be expected at all confidence levels with the exception of the $99.90 \%$. A possible explanation is that volatility was changing quickly during this time period while the parameters of the distributions were changing only once every year and so the parameters did not adequately reflect current market conditions. The moving window approach was slightly better than the static approach with the GL performing a little better than the GEV distribution. Overall, the only substantial advantage that accrued from the use of the GEV and GL distributions was the accurate prediction of the tail event at the $99.90 \%$ confidence level.

The distribution of the CRB index daily returns over the time period under examination was not very volatile (standard deviation was $0.60 \%$ ) with rather low skewness (-0.353) and kurtosis (2.234). As can be seen in Table 6, the VC model was seriously inaccurate at higher confidence levels regardless of the number of past returns used. For example, at the $99.75 \%$ confidence level the expected number of VaR violations was three but 11 to 14 were observed. The HS method tended to provide accurate VaR estimates at the $97.50 \%$ and $99.00 \%$ confidence levels when 250 and 500 past daily returns were used but this method did not capture all VaR excesses at the $99.90 \%$ confidence level regardless of the number of past returns used. The three VaR violations at the $99.90 \%$ confidence level when 1,000 past returns were used correspond to the negative returns of $-2.02 \%$ (May 18, 1987), -2.85\% (June 22, 1987) and -3.25\% (July 26, 1988) which can be very important if we consider that the CRB index is used as an underlying asset for futures and options contracts. The EWMA, on the other hand, was not particularly accurate at any confidence level. As can be expected, the performance of the MCS method was also poor, especially at high confidence levels which could be attributed to the inability of the normal distribution to provide good

\footnotetext{
${ }^{12}$ The 11 largest unexpected VaR violations from the VC250 were $-3.59 \%$ (May 15, 1987), $-3.54 \%$ (October 15, 1987), $-9.89 \%$ (October 19, 1987), -7.89\% (October 26, 1987), $-8.43 \%$ (October 28, 1987), -7.86\% (November 10, 1987), -6.24\% (October 16, 1989), -4.73\% (August 6,1990 ), $-3.23 \%$ (August 20, 1990), $-3.62 \%$ (August 21, 1990) and $-6.77 \%$ (August 19, 1991).
} 


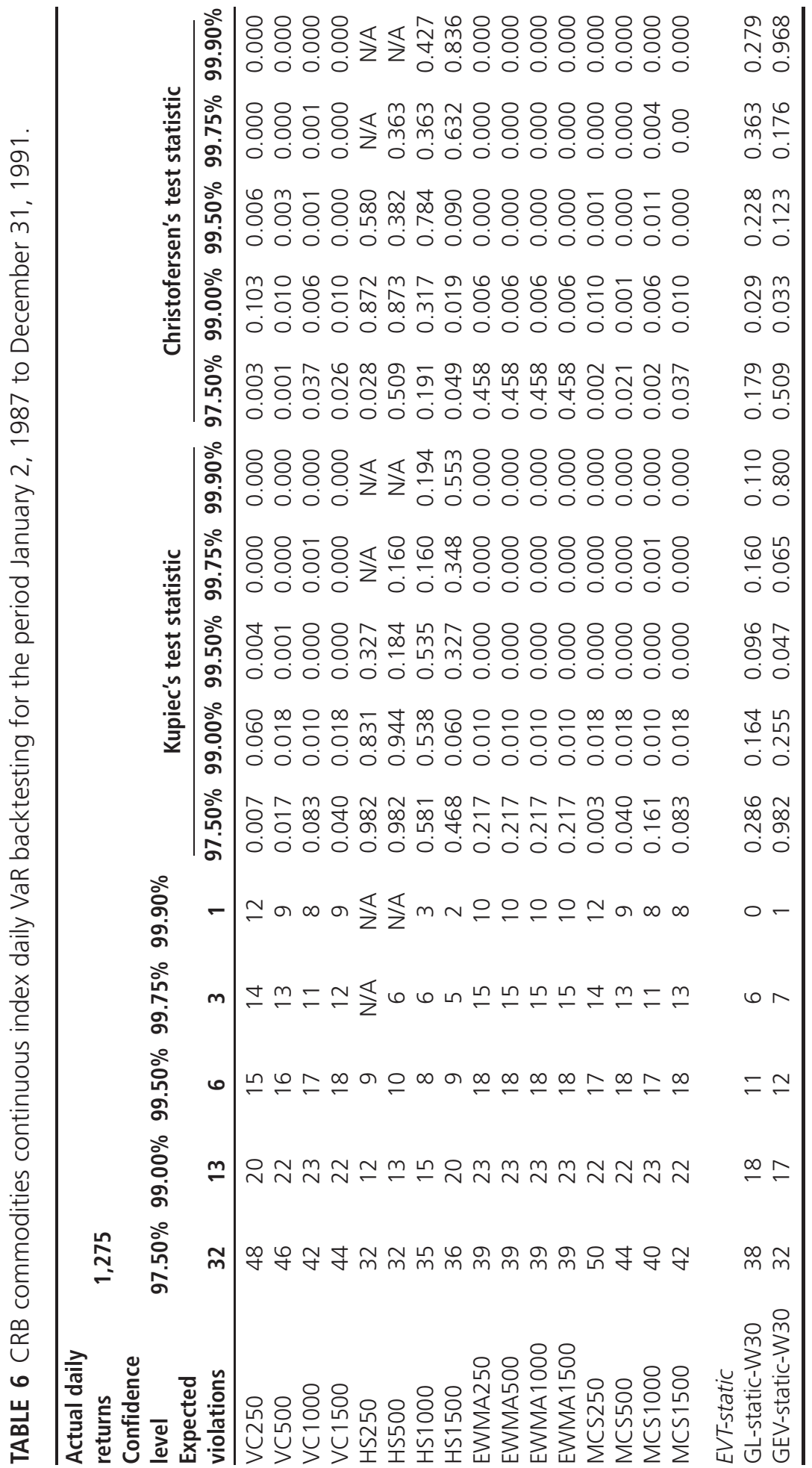




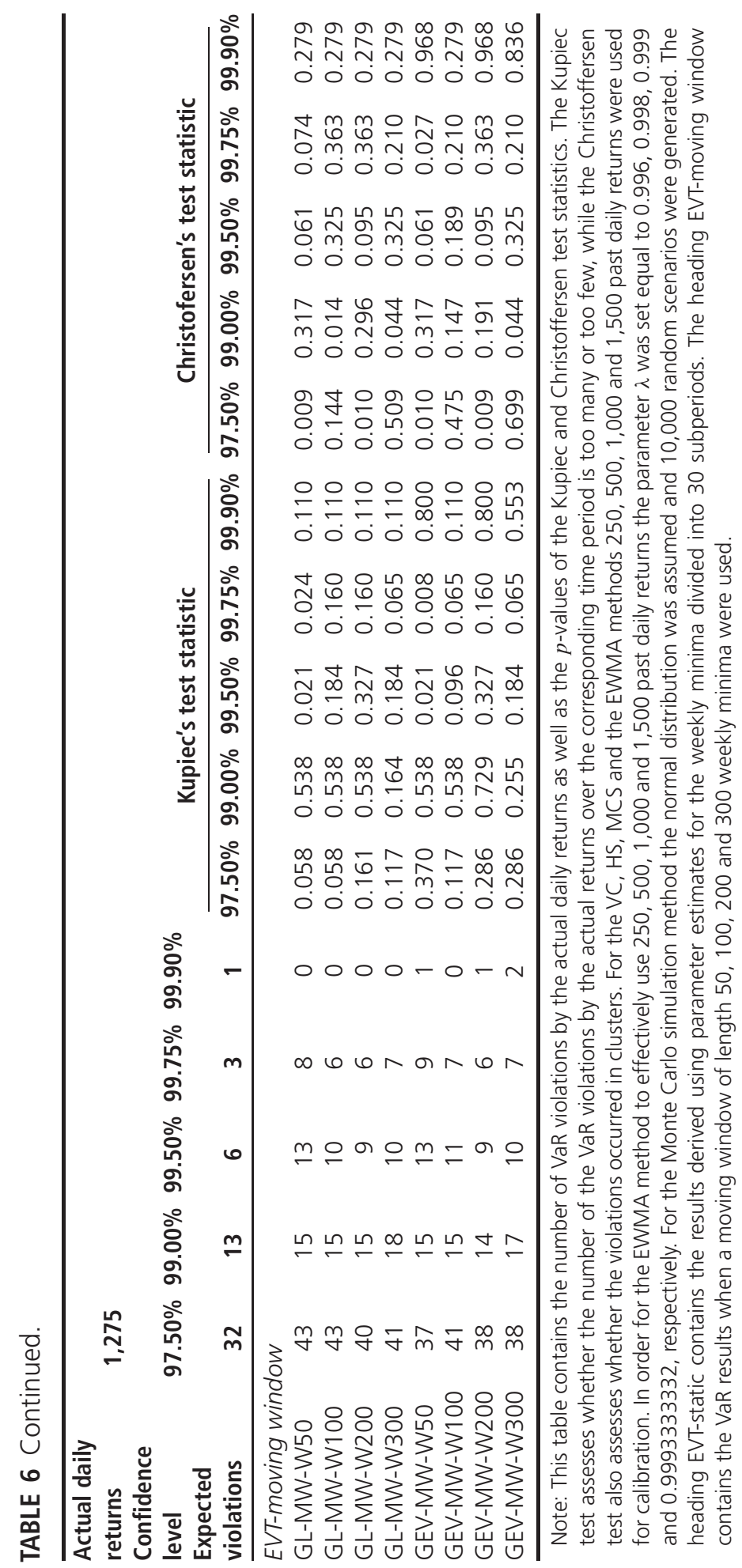


descriptions of the daily returns distribution. The performance of the GEV and GL distributions based on either the static or moving window approaches was accurate only at the $99.90 \%$ confidence level since at all other confidence levels the number of VaR violations were greater than expected.

An examination of Table 7, which contains the VaR results for the German bond, reveals similar patterns with respect to the ability of the VaR models used in this study to provide accurate VaR forecasts. In particular, the VC method is inaccurate at all confidence levels except the $99.00 \%$, where $13 \mathrm{VaR}$ violations were expected and the VC provided 12 to 14 . However, the low $p$-values of the Christoffersen test statistic imply that these violations occurred in clusters. The HS performs rather well deep into the tails of the returns distribution where only one violation occurred; however, at least 1,000 past returns are needed for such an accurate prediction. The EWMA method also performed rather well at the $99.90 \%$ but it was very inaccurate at the other confidence levels. The MCS was again very inaccurate at all confidence levels mainly due to the inaccurate assumption that daily returns follow a normal distribution. Probably, the most accurate VaR predictions of the extreme events came from the EVT method. In particular, the events that correspond to the $99.75 \%$ and $99.90 \%$ confidence levels were predicted quite well by both the GL and GEV distributions, with the GL being slightly better at the $99.75 \%$ confidence level.

In summary, EVT-based VaR estimates were found to be more accurate at high confidence levels compared with methods that assume that returns are normally distributed. The only other method which performed well was the HS; however, for estimates deep into the tail of the returns distribution, data availability might be an issue. At low confidence levels, EVT-based VaR did not offer any benefits over less sophisticated methods but this is to be anticipated since EVT focuses on the tails of the returns distribution and not its central part.

\section{IMPLICATIONS FOR REGULATORS AND FINANCIAL INSTITUTIONS}

The fundamental objectives of financial regulators and financial institutions are quite different. Regulators are mainly interested in reinforcing the stability of the financial system and, therefore, would tend to favor the most conservative VaR model: the one which results in the highest MCR. On the other hand, the profitability of financial firms is directly linked to the use of VaR models since the MCR is non-investable capital. Therefore, as Danielson et al (2001) argued, investment banks have incentives to favor VaR models that result in lower MCR, thus exposing financial firms to the really ruinous events located deep into the tail of the returns distribution. According to the Basel Committee on Banking Supervision (1996), a financial institution can choose between the standard approach proposed by the Bank for International Settlements (BIS) and their internal VaR model when calculating MCR. However, for those who choose to use in-house models, regulators require that VaR estimates should be multiplied by a factor of at least three. Based on the backtesting evaluation of a bank's VaR model, BIS may increase the multiplier further by an increment between 


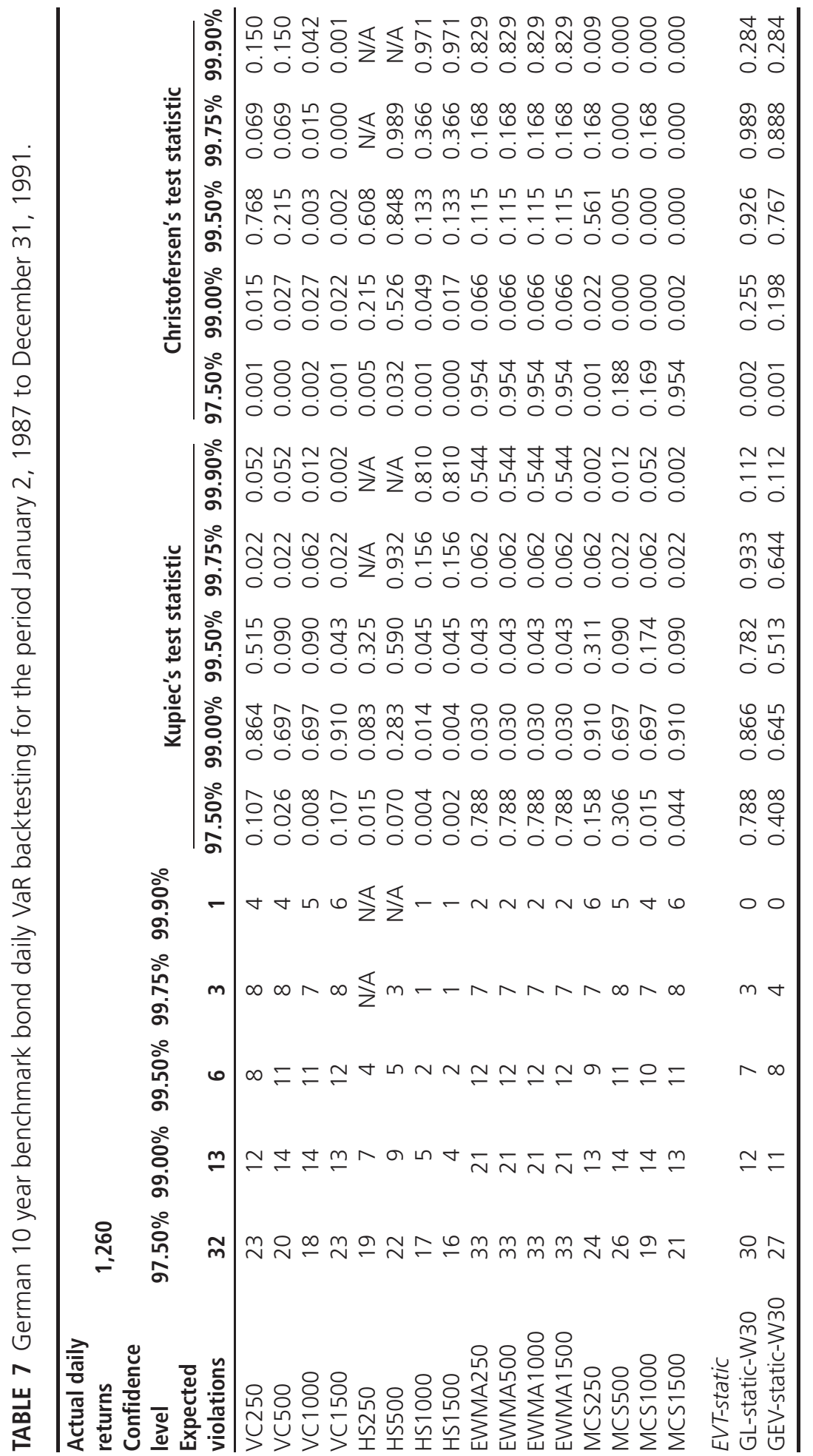




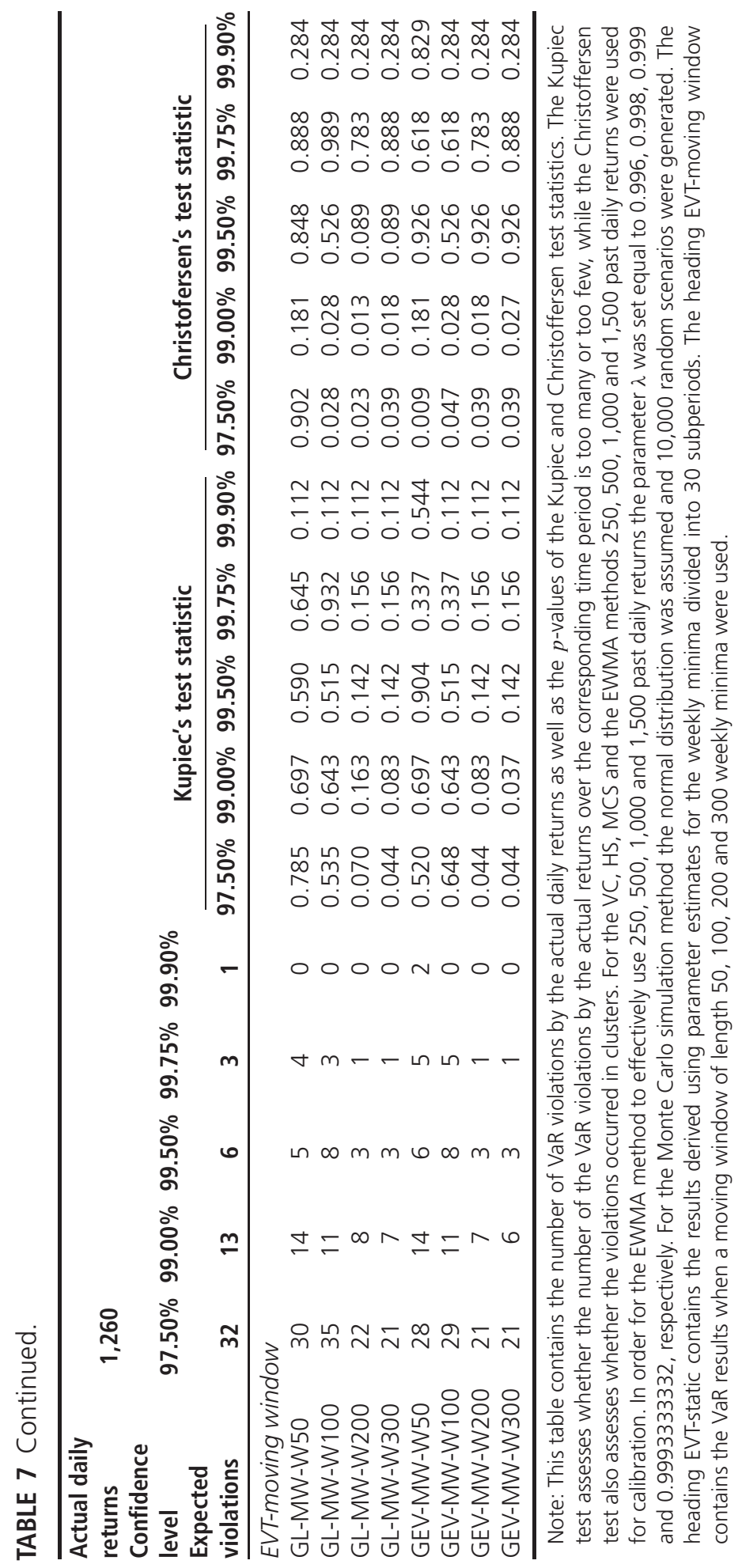


zero and one. This rule has been criticized by many researchers (Longin (2000); Danielson et al (1998)) as being too crude giving rise to high MCR values; thus it eliminates any incentives that banks might have to improve their internal models. According to the standard approach, the MCR of an equity position must be at least $12 \%$ of the position and aims to cover the maximum loss over a period of 10 days. ${ }^{13}$ Thus, approximately, dividing by the square root of 10 we could derive the daily capital charge: that is $3.79 \%$.

Table 8 contains the daily VaR estimates for the CAC-DS, CRB and German bond indexes daily returns on the October 19, 1987, July 26, 1988 and October 20, 1987, respectively, which are the dates where the minimum daily returns occurred $(-9.89 \%,-3.25 \%$ and $-5.23 \%$ for the CAC-DS, CRB and German bond indexes, respectively). Clearly, in the case of the CAC-DS index, the capital charge of $3.79 \%$ is much less than the actual loss of $-9.89 \%$ implying that the standard approach of BIS offers inadequate coverage against extreme events. On the other hand, the use of EVT can provide far better predictions against these rare market movements. For example, the VaR estimate at the $99.90 \%$ confidence level provided by the GL-MW-W100 is $-9.33 \%$, which is very close to the actual daily return of $-9.89 \%$. The best prediction derived using traditional methods was $-5.97 \%$ by the HS1000 and HS1500; however, a far from adequate estimation. However, if a bank was to multiply the VaR estimates derived by EVT by a factor between three and four, the capital charges would be enormous (eg, between $29 \%$ and $37 \%$ ). Therefore, although EVT can provide accurate tail predictions, the use of the multiplication factor will make MCRs very high, thus deterring banks from considering its use.

When the VaR estimates for the CRB index on July 26, 1988 are examined a quite different picture emerges. In particular, it appears that the EVT methods either underestimates or overestimates risk. For example, the VaR estimates given by the GL-MW-W50 method are $-2.75 \%$ at the $99.75 \%$ confidence level and $-6.09 \%$ at the $99.90 \%$ confidence level. This is probably because the distribution of the CRB index daily returns was not fat tailed during that period; in fact the kurtosis was 2.234. Consequently, other methods appear to provide better estimates of the extreme event (eg, the EWMA at the $99.75 \%$ confidence level). In the case of the German bond index, the daily return that occurred on October 20, 1987 was $-5.23 \%$ while the empirical distribution of returns was almost symmetrical but with a kurtosis value of 6.418 . It can be noticed that none of the traditional VaR methods provided an accurate forecast of the actual return. The EVT based VaR estimates tended to underestimate risk by a smaller amount at the $99.75 \%$ confidence level compared to the traditional

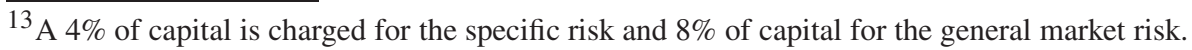
The BIS defined specific risk to be the gross equity position in the market as a whole (the sum of all long and all short equity positions) and general market risk to be the net equity position (the difference between the sum of the long and the sum of the short equity positions). The capital charge for specific risk is $8 \%$ but if the portfolio is well diversified and liquid reduces to $4 \%$. The indexes used in this paper can be considered to be both well diversified and liquid portfolios, therefore a capital charge of $4 \%$ for specific risk is assumed.
} 


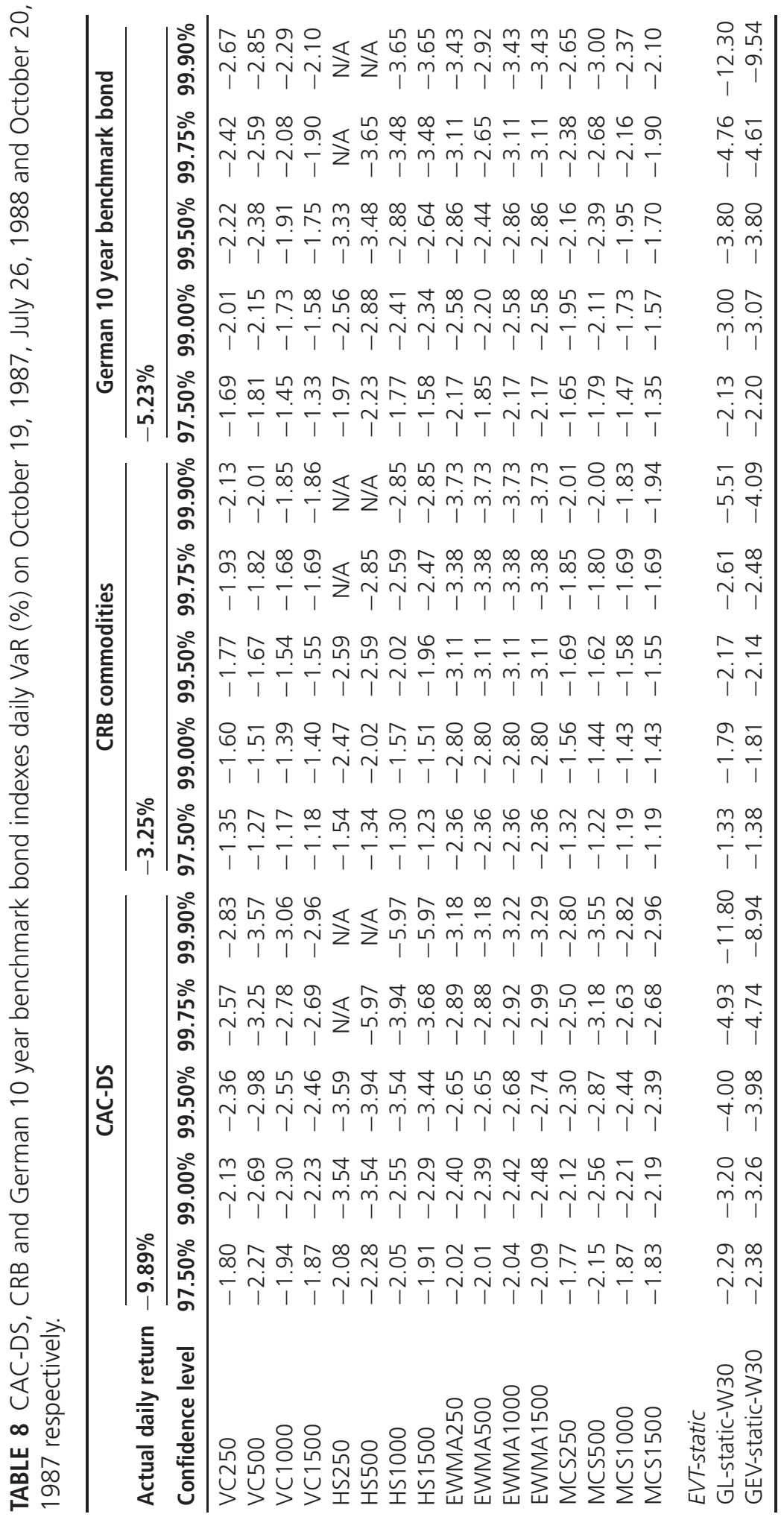




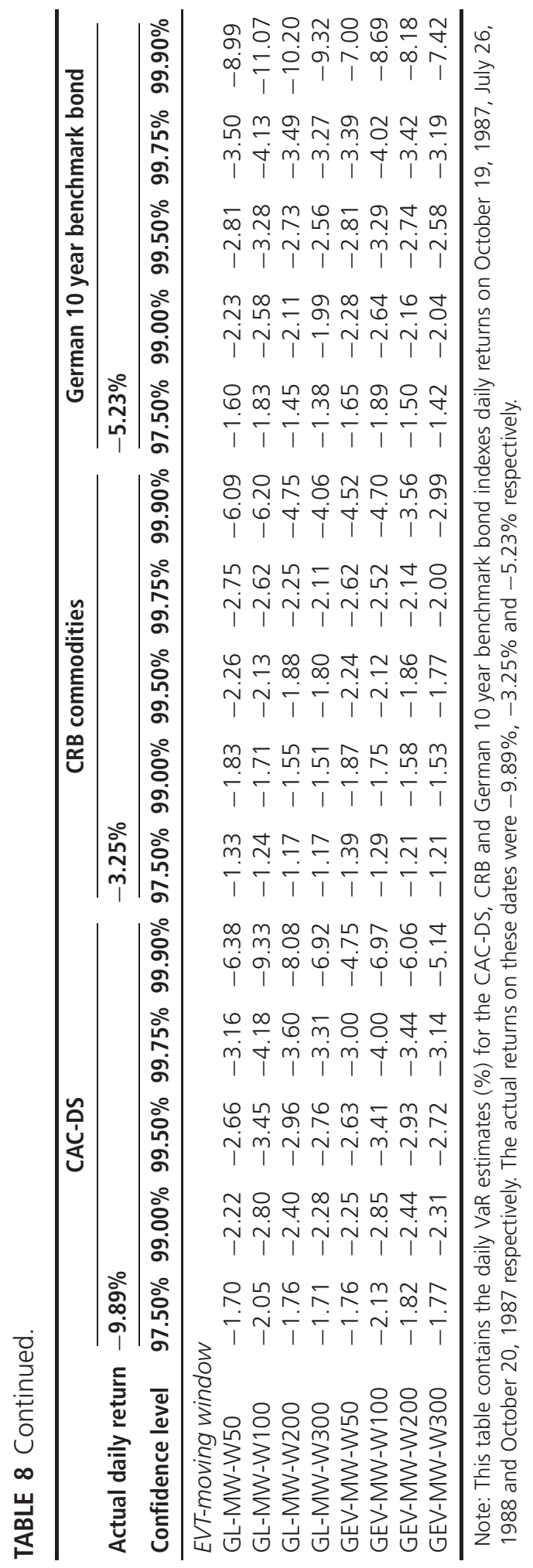




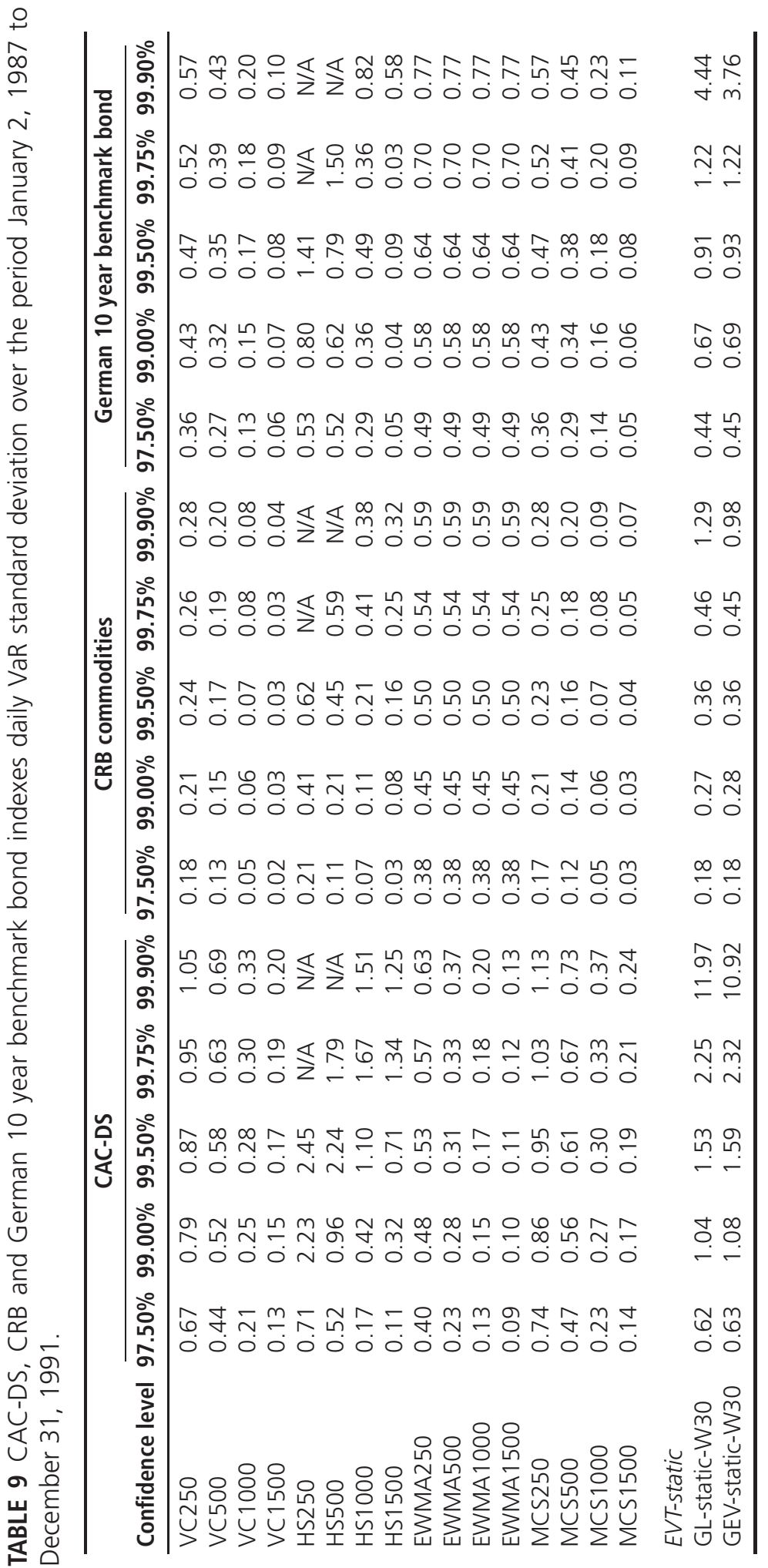




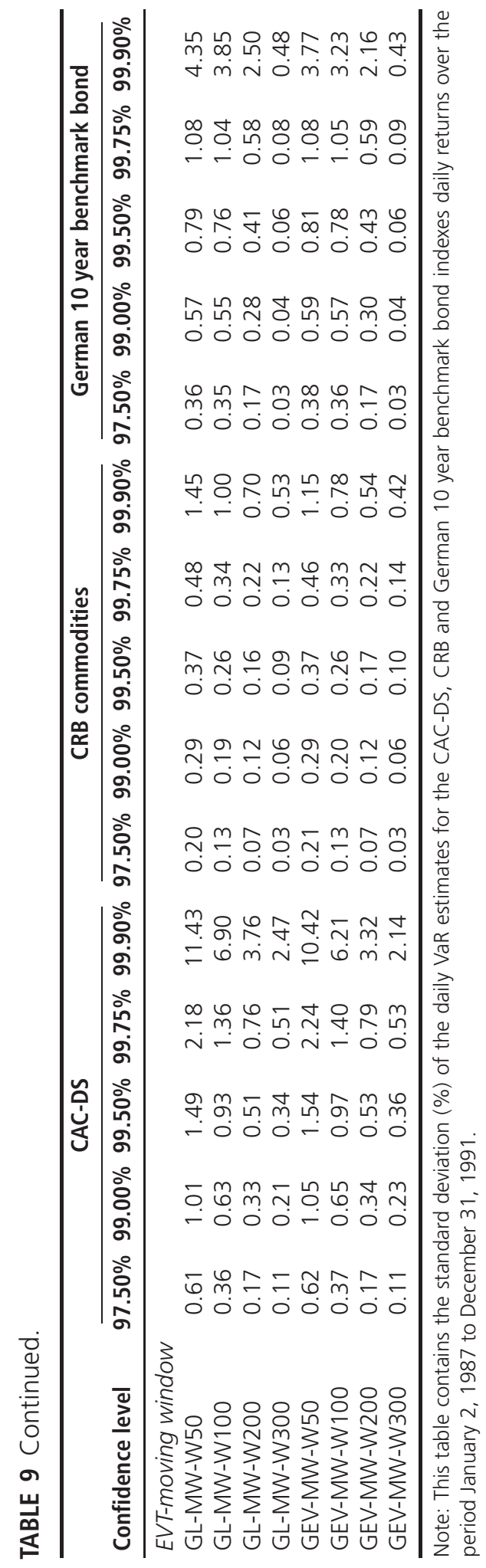


methods while they tended to overestimate risk at the high confidence level of $99.90 \%$. For example, at the $99.75 \%$ confidence level the closer VaR estimate to the actual return was provided by the GL-static-W30 method $(-4.76 \%)$ while at the $99.90 \%$ confidence level the best estimate was provided by the GEVMW-W50 method (-7.00\%). Despite the overestimation of risk, these EVT based VaRs would provide an adequate coverage against catastrophic losses. However, applying the BIS multiplication factor would render these VaR estimates extremely conservative leading to huge MCR.

In addition, Jorion (2002) argued that financial institutions would tend to favor $\mathrm{VaR}$ models that generate estimates of low variability because they would not be forced to sell assets or change their trading strategies frequently in order to satisfy regulatory requirements. Table 9 contains the standard deviation of the VaR estimates during the time period January 2, 1987 to December 31, 1991. The variability of the EVT based VaR is, in general, similar to the variability of VaR provided by the other methods. However, at the $99.90 \%$ confidence level, the EVT-based VaR estimates are much more volatile. It can also be noticed that the standard deviation of the CAC-DS index VaR at the $99.90 \%$ confidence level is much higher than those of the CRB and German bond indexes. This feature can probably be attributed to the fatter-tailed distribution of the CAC-DS index daily returns. Taking into account, therefore, the objectives of a financial institution and the volatile and relatively large VaR values that the EVT method provides at the 99.90\% confidence level, it could be argued that a financial institution would be reluctant to adopt EVT analysis in VaR modeling unless the multiplication factor was to be reduced or even abolished.

\section{CONCLUSION}

In this paper EVT methods were used to derive VaR estimates related to the lower tail of the daily returns of the CAC-DS, CRB and German bond indexes. The analysis of extremes revealed that the too much celebrated GEV distribution is not the best model for the extreme minima of the daily returns since a fattertailed distribution, the GL, offers better descriptions. Considering that current applications of EVT in finance focus on either the GEV or GP distributions the implication is that the probabilities of the ruinous extreme events may be underestimated. The results also indicated that the behavior of extremes is time variant and it is affected by economic and political events.

With respect to VaR, the empirical results indicated that EVT methods can be valuable when the interest is in protecting a portfolio from the really catastrophic events located deep in the lower tail of the returns distribution. At low confidence levels, however, EVT based VaR did not offer any benefits over less sophisticated methods but this was to be expected since EVT focuses on modeling the tails of the returns distribution and not its central part. EVT based VaR estimates were also found to be larger than those derived by traditional methods, leading to higher MCR. In that respect, one could argue that the BIS multiplication factor is too high, thus discouraging financial institutions from adopting EVT methods when 
deriving VaR. The results also showed that techniques which capture some of the time variant nature of the extremes distribution have the potential to improve the accuracy of VaR estimates since current market conditions are explicitly taken into account.

\section{APPENDIX A}

The GEV and GL are three parameter distributions which have cdfs, quantile functions and parameter estimates as shown in Table A.1. The parameters $\kappa, \alpha$ and $\beta$ are called the shape, scale and location, respectively.

\section{TABLE A.1}

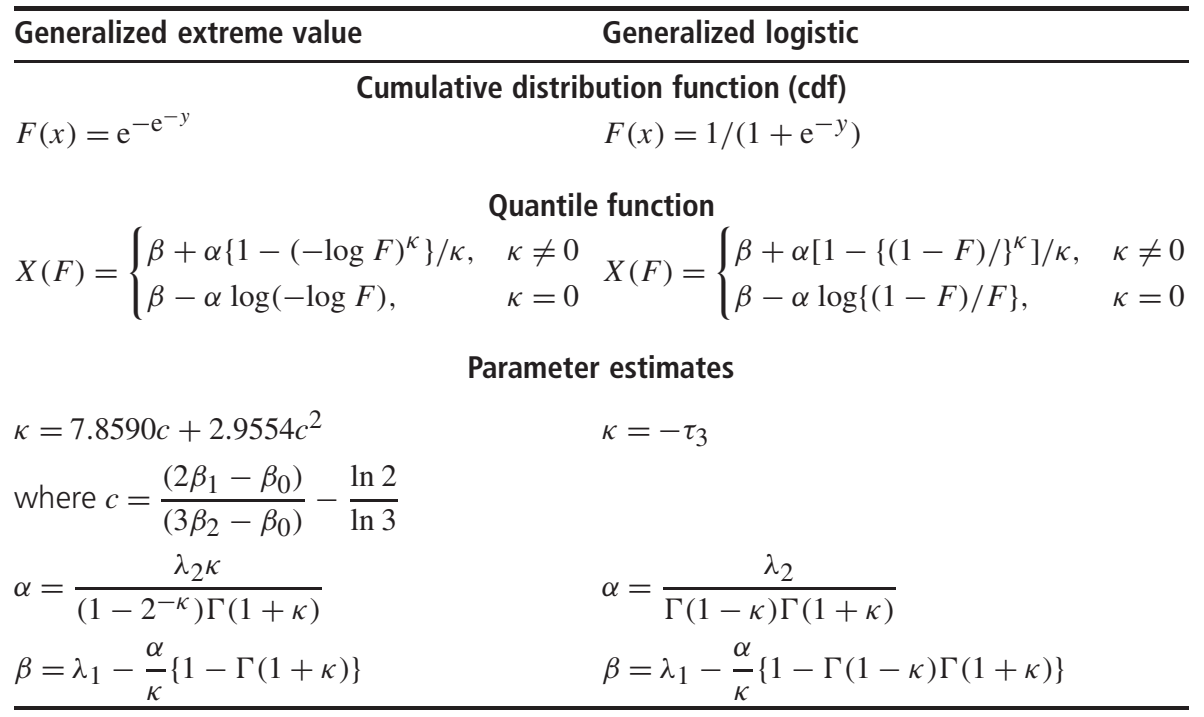

\section{REFERENCES}

Anderson, T. W., and Darling, D. A. (1954). A test for goodness of fit. The American Statistical Association 49(268), 765-769.

Aparicio, F. M., and Estrada, J. (2001). Empirical distributions of stock returns: European securities markets, 1990-95. European Journal of Finance 7(1), 1-21.

Bali, T. G. (2003). An extreme value approach to estimating volatility and value at risk. Journal of Business 76(1), 83-108.

Bali, T. G., and Neftci, S. N. (2003). Disturbing extremal behavior of spot rate dynamics. Journal of Empirical Finance 10, 455-477.

Bali, T. G., and Weinbaum, D. (2005). A conditional extreme value volatility estimator based on high frequency returns. Journal of Economic Dynamics and Control 31(2), 361-397.

Balkema, A., and de Haan, L. (1974). Residual time life at great age. Annals of Probability 2, 792-804. 
Basel Committee on Banking Supervision (1996). Amendment to the Capital Accord to Incorporate Market Risks. Bank for International Settlements, Basel.

Byström, H. N. E. (2004). Managing extreme risk in tranquil and volatile markets using conditional extreme value theory. International Review of Financial Analysis 13(2), 133-152.

Choulakian, V., and Stephens, M. A. (2001). Goodness-of-fit tests for the generalized Pareto distribution. Technometrics 43(4), 478-484.

Christoffersen, P. F. (1998). Evaluating interval forecasts. International Economic Review 39(4), 841-862.

Coles, S. (2001). An Introduction to Statistical Modeling of Extreme Values (Springer Series in Statistics). Springer, London.

Cotter, J., and Dowd, K. (2006). Extreme spectral risk measures: an application to futures clearinghouse margin requirements. Journal of Banking and Finance 30(12), 34693485 .

d'Agostino, B., and Stephens, M. A. (1986). Goodness of Fit Techniques. Marcel Dekker, New York.

Da Silva, A. L. C., and de Melo Mendes, B. V. (2003). Value-at-risk and extreme returns in Asian stock markets. International Journal of Business 8(1), 17-40.

Danielson, J. (2002). The emperor has no clothes: limits to risk modelling. Journal of Banking and Finance 26(7), 1273-1296.

Danielson, J., Embrechts, P. L., Goodhart, C., Keating, C., Munnich, F., Renault, O., and Shin, H. S. (2001). An academic response to Basel II. Financial Markets Group, Special Paper, No. 130, London School of Economics.

Danielson, J., Hartmann, P., and de Vries, G. C. (1998). The cost of conservatism. Risk 11(1), 103-107.

Embrechts, P., Kluppelberg, C., and Mikosh, T. (1999). Modelling Extremal Events for Insurance and Finance, 2nd edn. Springer-Verlag, Berlin.

Engle, R. F., and Manganelli, S. (2004). CAViaR: conditional autoregressive value at risk by regression quantiles. Journal of Business and Economic Statistics 22(4), 367-381.

Fisher, R. A., and Tippet, L. H. C. (1928). Limiting forms of the frequency distribution of largest or smallest member of a sample. Proceedings of the Cambridge Philosophical Society 24, 180-190.

Gençay, R., and Selçuk, F. (2004). Extreme value theory and value-at-risk: relative performance in emerging markets. International Journal of Forecasting 20(2), 287-303.

Gettinby, G. D., Sinclair, C. D., Power, D. M., and Brown, R. A. (2004). An analysis of the distribution of extremes share returns in the UK from 1975 to 2000. Journal of Business Finance and Accounting 31(5), June, 607-646.

Gilli, M., and Këllezi, E. K. (2006). An application of extreme value theory for measuring financial risk. Computational Economics 27(1), 1-23.

Gnedenko, B. V. (1943). Sur la distribution limited du terme maximum d'une série aléatoire. Annals of Mathematics 44(3), 423-453. 
Hill, B. M. (1963). The three parameter lognormal distribution and Bayesian analysis of a point source epidemic. Journal of the American Statistical Association 58(301), 72-84.

Hosking, J. R. M. (1990). L-moments: analysis and estimation of distributions using linear combinations of order statistics. Journal of the Royal Statistical Society, Series B 52(1), 105-124.

Hosking, J. R. M., and Wallis, J. R. (1987). Parameters and quantile estimation for the generalised Pareto distribution. Technometrics 29(3), 339-349.

Hosking, J. R. M., Wallis, J. R. and Wood, E. F. (1985). Estimation of the generalised extreme-value distribution by the method of probability-weighted moments. Technometrics 27(3), 251-261.

Jondeau, E., and Rockinger, M. (2003). The tail behaviour of stock returns: emerging versus mature markets. Journal of Empirical Finance 10(5), 559-581.

Jorion, P. (2002). Fallacies about the effects of market risk management systems. Bank of England. Financial Stability Review (13), 115-127.

Kearns, P., and Pagan, A. (1997). Estimating the tail index for financial time series. Review of Economics and Statistics 79(2), 171-175.

Kupiec, P. H. (1995). Techniques for verifying the accuracy of risk measurement models. Journal of Derivatives 3, 73-84.

Landwehr, J. M., Matalas, N. C., and Wallis, J. R. (1979). Probability weighted moments compared with some traditional techniques in estimating Gumbel parameters and quantiles. Water Resources Research 15, 1055-1064.

Leadbetter, M. R., Lindgren, G., and Roótzen, H. (1983). Extremes and Related Properties of Random Sequences and Processes. Springer, New York.

Longin, F. M. (1996). The asymptotic distribution of extreme stock market returns. Journal of Business 69(3), 383-408.

Longin, F. M. (2000). From value at risk to stress testing: the extreme value approach. Journal of Banking and Finance 24(7), 1097-1130.

Lux, T. (2001). The limiting extremal behaviour of speculative returns: an analysis of intra-daily data from the Frankfurt stock exchange. Applied Financial Economics 11(3), 299-315.

McNeil, A. J., and Frey, R. (2000). Estimation of tail-related risk measures for heteroscedastic financial time series: an extreme value approach. Journal of Empirical Finance 7(3\&4), 271-300.

Neftci, S. N. (2000). Value at risk calculations, extreme events, and tail estimation. Journal of Derivatives 7(Spring), 23-38.

Pickands, J. I. (1975). Statistical inference using extreme order statistics. Annals of Statistics 3, 119-131.

Pownall, R. A. J., and Koedijk, K. G. (1999). Capturing downside risk in financial markets: the case of the Asian crisis. Journal of International Money and Finance 18(6), 853870.

Roótzen, H., and Tajvidi, N. (1997). Extreme value statistics and wind storm losses: a case study. Scandinavian Actuarial Journal 97, 70-94. 
Sankarasubramanian, A., and Srinivasan, K. (1999). Investigation and comparison of sampling properties of L-moments and conventional moments. Journal of Hydrology 218(1\&2), 13-34.

Smith, R. L. (1987). Estimating tails of probability distribution. Annals of Statistics 15(3), 1174-1207.

Stephens, M. A. (1976). Asymptotic results for goodness-of-fit statistics with unknown parameters. Annals of Statistics 4(2), 357-369.

Tolikas, K., and Brown, R. A. (2006). The distribution of extreme daily share returns in the Athens stock exchange. European Journal of Finance 12(1), 1-22.

Vogel, R. M., and Fennessey, N. M. (1993). L-moment diagrams should replace productmoment diagrams. Water Resources Research 29(6), 1745-1752. 